Portland State University

PDXScholar

Civil and Environmental Engineering Faculty

Publications and Presentations

2-1-2019

\title{
Maximum Coverage Capacitated Facility Location Problem with Range Constrained Drones
}

\author{
Darshan Chauhan \\ Portland State University, drc9@pdx.edu \\ Avinash Unnikrishnan \\ Portland State University, uavinash@pdx.edu \\ Miguel Figliozzi \\ Portland State University, figliozzi@pdx.edu
}

Follow this and additional works at: https://pdxscholar.library.pdx.edu/cengin_fac

Part of the Civil and Environmental Engineering Commons

Let us know how access to this document benefits you.

\section{Citation Details}

Chauhan, D., Unnikrishnan, A., \& Figliozzi, M. (2019). Maximum coverage capacitated facility location problem with range constrained drones. Transportation Research: Part C, 99, 1-18.

This Post-Print is brought to you for free and open access. It has been accepted for inclusion in Civil and Environmental Engineering Faculty Publications and Presentations by an authorized administrator of PDXScholar. Please contact us if we can make this document more accessible: pdxscholar@pdx.edu. 


\title{
Maximum Coverage Capacitated Facility Location Problem with Range Constrained Drones
}

\author{
Darshan Chauhan $^{\mathrm{a}}$, Avinash Unnikrishnan ${ }^{\mathrm{a}, *}$, Miguel Figliozzi ${ }^{\mathrm{a}}$ \\ ${ }^{a}$ Department of Civil and Environmental Engineering, Portland State University, OR 97201
}

\begin{abstract}
Given a set of demand and potential facility locations and a set of fully available charged drones, an agency seeks to locate a pre-specified number of capacitated facilities and assign drones to the located facilities to serve the demands. The facilities serve as drone launching sites for distributing the resources. Each drone makes several one-to-one trips from the facility location to the demand points and back until the battery range is met. The planning period is short-term and therefore the recharging of drone batteries is not considered. This paper presents an integer linear programming formulation with the objective of maximizing coverage while explicitly incorporating the drone energy consumption and range constraints. The new formulation is called the Maximum Coverage Facility Location Problem with Drones or simply MCFLPD. The MCFLPD is a complex problem and even for relatively small problem sizes a state of the art MIP solver may require unacceptably long running times to find feasible solutions. Computational efficiency of MCFLPD solutions is a key factor since conditions associated with customer demands or weather conditions (e.g., wind direction and speed) may change suddenly and require a fast global reoptimization. To better balance solution quality and running times novel greedy and three-stage heuristics (3SH) are developed. The 3SH is based on decomposition and local exchange principles and involves a facility location and allocation problem, multiple knapsack subproblems, and a final local random search stage. On average the $3 \mathrm{SH}$ solutions are within $5 \%$ of the best Gurobi solutions but at a small fraction of the running time. Multiple scenarios are run to highlight the importance of changes in drone battery capabilities on coverage.
\end{abstract}

Keywords: UAV, Drones, Maximum Coverage Facility Location, Greedy and Decomposition Heuristics, Energy, range and capacity constraints

\section{Introduction}

Several companies like Amazon, Google, UPS, and Flytrex are evaluating the potential use of Unmanned Aerial Vehicles (UAVs) or drones for commercial service or package deliveries (Mack, 2018). Drones are not restricted by the availability of existing infrastructure and therefore can lead to improved last-mile efficiency, safety, and reliability (DHL, 2014). Drones are particularly suitable for emergency applications like search and rescue (Karaca et al., 2018), deliveries of critical medical supplies post-disaster or for emergency response

\footnotetext{
*Corresponding author
} 
(Thiels et al., 2015; Scott and Scott, 2018), and crop irrigation and pesticide spraying (Albornoz and Giraldo, 2017; Berner and Chojnacki, 2017; Burema and Filin, 2016; Wang et al., 2016; Giles et al., 2016; Faiçal et al., 2014; Costa et al., 2012). Advances in drone technologies regarding lighter and stronger materials for frames (Hassanalian and Abdelkefi, 2017), sensing and coordinating algorithms (Yanmaz et al., 2018, 2017), battery capacity (Li et al., 2017; Fehrenbacher, 2018) and a predictable regulatory framework (FAA, 2018b) are expected to accelerate large-scale UAV adoption. However, a key challenge to drone deliveries is their limited range and payload.

Drones have significantly smaller payload carrying capacities and ranges compared to trucks. A diesel cargo van RAM Pro Master 2500 has 378 times the carrying capacity and nearly 20 times the range of a typical drone (Figliozzi, 2017). Moreover, the maximum range of the drone decreases as payload increases. When delivery locations are distributed across a region (urban or rural) trucks can usually cover all demand points from one depot. However, due to its limited range, a drone-based delivery system requires more depots or launching sites distributed across the region. Also, unexpected and/or adverse weather conditions, e.g., headwinds, may dramatically alter the energy consumption and/or range of a delivery drone. Hence, computational efficiency is vital when analyzing drone delivery systems since conditions associated with customer demands and/or weather conditions (e.g. wind direction and speed) may change suddenly and require a fast global reoptimization. A contribution of this research is a novel integer programming model to locate drone launching facilities to meet the demands of spatially distributed customers. This model is called the Maximum Coverage Facility Location Problem with Drones (MCFLPD) and comprises the following: (i) selection of a pre-specified number of capacitated facilities from a list of potential facility locations as drone launching sites, (ii) distribution of a limited number of drones to the selected facilities, (iii) assignment of demand locations to open facilities and drones while respecting the capacity of the facility and the range constraints of the drones.

One of the motivations behind the choice of coverage objective is to evaluate the feasibility of using drones to deliver medical supplies such as defibrillators (Boutilier et al., 2017; Claesson et al., 2017), blood deliveries (Amukele et al., 2017) or critical relief after extreme natural events (Anaya-Arenas et al., 2014; HolguínVeras et al., 2012; Ozdamar, 2011) while accounting for drone battery range limitations. To better balance solution quality and running times novel greedy and a three-stage heuristics (3SH) are developed. The 3SH is based on decomposition and local exchange principles and involves a facility location and allocation problem, multiple knapsack subproblems, and a final exchange based random search stage.

After a literature review section, the MCFLPD and the proposed solution heuristics are introduced. A real-world case study for making drone deliveries in Portland, OR is presented next. Results concerning solution quality and running times are later presented and discussed. The paper ends with battery sensitivity analyses and conclusions. 


\section{Literature Review}

A majority of the research on drone delivery applications have focused on UAV or drone routing and scheduling leading to several interesting variants of the traveling salesman and vehicle routing problems. Murray and Chu (2015) studied the flying sidekick traveling salesman problem (FSTSP) where a drone and a truck deliver in collaboration to a set of customers. The drone takes-off from the truck, makes the delivery, and rendezvous back with the truck at a different location. Murray and Chu (2015) also proposed the parallel drone scheduling traveling salesman problem (PDSTSP) where a set of UAVs and a truck make deliveries from a single depot to customers. Murray and Chu (2015) provide mixed integer linear programming formulations and a route and reassign heuristic for solving the FSTSP and a partitioning heuristics for solving the PDSTSP problem. Ponza (2016) modified the drone delivery time constraints in Murray and Chu (2015)'s FSTSP formulation and developed a simulated annealing metaheuristic. Agatz et al. (2018) denoted the FSTSP as Traveling Salesman Problem with Drones (TSPD), provided approximation results comparing TSPD and TSP optimal solution, and developed several route-first cluster second heuristics which vary in the initial tour generation and assignment of drone delivery nodes. Yurek and Ozmutlu (2018) solved the TSPD using a two-stage iterative decomposition approach where truck routes are determined in the first stage, and drone nodes are assigned in the second stage. Ha et al. (2018) focused on the min-cost TSPD variant of Murray and Chu (2015)'s FSTSP and developed a greedy randomized adaptive search procedure which builds TSPD routes from TSP routes. Carlsson and Song (2017) applied a continuous approximation to the FSTSP (denoted as horsefly routing problem in this paper) and proved that the efficiency gain by adding a drone is a function of the square root of the ratio of the drone and truck velocities.

Wang et al. (2017); Poikonen et al. (2017) developed several worst-case bounds for the vehicle routing problem with drones (VRPD) where several delivery trucks and drones (launched from trucks) are used to satisfy demands. The bounds provide insights on modifying existing solution algorithms for TSP and VRP variants to obtain solutions to VRPD. Daknama and Kraus (2017) found several nested local search heuristics to be more efficient than the greedy drone assignment approach in solving VRPD. Dayarian et al. (2017) studied the vehicle routing with drone resupply problem where a single drone resupplies a delivery truck from a depot and found that the use of drones improved delivery reliability. Dayarian et al. (2017) studied the dynamic and multiple vehicles and drones variant of Murray and Chu (2015)'s PDSTSP. The authors developed an approximate dynamic programming based heuristic decision making policy to spatially

partition the customers into those being served by trucks and those being served by drones. The results show that adding drones to truck fleets can reduce fleet size and increase deliveries. In contrast to the above works, we consider a drone only delivery system and do not consider truck deliveries as this is the case for medical supplies (Amukele et al., 2017).

Dorling et al. (2017) modeled the drone delivery problem as a single depot multi-trip vehicle routing problem and used linear approximations to study the impact of battery and payload weight on energy consumption. The model was solved using a simulated annealing metaheuristic. Optimizing battery weight 
was found to be critical for system efficiency. Kim et al. (2018) use a robust optimization approach to model the impact of air temperature uncertainty on drone battery capacity and studied the ability of a fleet of drones to visit multiple locations. Choi and Schonfeld (2017) used a continuous approximation approach to understand the factors affecting a fleet of drone delivery systems. The authors found that battery improvements are critical to overall system coverage and drone delivery systems are more effective in areas with higher demand densities. In this work, we do not model one-to-many deliveries on each route. We assume that the drones make multiple one-to-one deliveries from the depot locations subject to battery range constraints as is the case with current deliveries of blood supplies.

Recently, several researchers have focused on facility location problems for drone delivery systems that are more closely related to the topic of this research. For example, Chowdhury et al. (2017) used a continuous approximation approach to develop a humanitarian logistics supply chain post-disaster considering both drones and truck deliveries. The objective is to minimize transportation, inventory, and facility location costs. We adopt a discrete approach with the objective of maximizing coverage. Golabi et al. (2017) studied the relief distribution center location model post-disaster where edges may or may not have collapsed due to disaster. Inaccessible demand points are served using drones. The objective is to minimize the travel times of demand points to located facilities and travel time from facilities to inaccessible drones. The model was solved using several metaheuristics with genetic algorithm being the most efficient. Pulver and Wei (2018) developed a facility location model to maximize primary and secondary coverage in the context of transporting and delivering medical supplies using drones. Pulver and Wei (2018) do not consider capacity constraints at drone launching sites or energy consumption as a function of payload and distance for each individual delivery. Also, this paper considers range constraint on multiple trips whereas Pulver and Wei (2018) assume that only one trip is made in the planning period by a drone.

Kim et al. (2017) developed a two-stage model for drone-based pickup and deliveries of medical supplies. In the first stage, a set covering problem is solved to establish depot locations. The second stage is a multidrone vehicle routing problem. Both Pulver and Wei (2018) and Kim et al. (2017) use solvers such as Gurobi and GAMS to solve their models. This paper considers a single stage formulation for locating capacitated facilities and assigning demand points to drones. This research also models the allocation of a fixed amount of drones to facilities which is not considered in most of the works mentioned above.

Hong et al. (2018) study a drone recharging facility location problem which can help increase the coverage range of drones for commercial deliveries. The analysis is based on the worst case drone range at maximum payload. The model is solved using a multi-stage heuristic which embeds principles of greedy, exchange, and simulated annealing solution algorithms. Other researchers have focused on comparing drone delivery systems with traditional truck-based deliveries from an emission and sustainability perspective. Goodchild and Toy (2017) conduct a GIS-based simulation analysis and determine that factors such as distance from depot and number of recipients affect the relative $\mathrm{CO}_{2}$ emissions of UAVs versus trucks. The authors recommend a mixed drone truck delivery system. Figliozzi (2017) uses continuous approximation techniques 
and derive analytical formulas to compare operational and lifecycle emissions and energy consumptions of UAVs with conventional diesel, electric vans, and tricycle delivery services. Figliozzi (2017) shows that the delivery strategy (grouping of customers in a route) affects the relative $\mathrm{CO}_{2}$ emission efficiencies.

A substantial amount of literature exists on the maximum covering facility location problem (MCFLP) (Church and ReVelle, 1974). Farahani et al. (2012) and Daskin (2011) provide excellent reviews of different variants of MCFLP and associated solution strategies. The MCFLPD model considered in this work is a more complicated variant of MCFLP as the coverage is a function of the drone range which in turns depends on drone availability as well as the payload. The MCFLPD model also has similarities with the Capacity and Distance Constrained Plant Location Problem (Albareda-Sambola et al., 2009). While Albareda-Sambola et al. (2009) focus on minimizing cost, the model presented in this work focuses on maximizing coverage. We also explicitly model the distance range constraints resulting from the interaction between battery capacity and the demand carried in a trip. Also, Albareda-Sambola et al. (2009) assume a pre-specified number of trucks are available to each open facility whereas, in our model, we assume that a pre-specified number of drones are available to the entire system. The additional drone allocation feature adds to the complexity of the formulation.

Otto et al. (2018) provide a detailed review of all optimization based papers on civil applications of drones and UAVs. To the best of the authors' knowledge, the MCFLPD model studied in this paper is a novel contribution and the first research to explicitly include drone energy consumption as a function of payload and distance within a drone maximum coverage location problem framework. The solution approaches, the case study, and the sensitivity analysis are also novel contributions.

\section{Problem Formulation}

This section presents the integer linear formulation for the MCFLPD. At the beginning of the planning period, an agency is given a set of demand locations $I$ each having demand $w_{i}$, a set of potential facility locations $J$, and set of available fully-charged drones $K$. The planning period or time period of analysis is short-term and will depend on the application (for delivery of blood supplies post-earthquake maybe six hours; for delivery of medicine/food in case of an earthquake maybe one day). The agency's goal is to locate $p$ facilities to maximize the demand served. The agency will allocate resource of mass $U$ to each located facility representing the maximum amount of demand which can be served by each located facility in a planning period. $U$ can be viewed as the capacity of the facility. The capacity of a facility corresponds to the maximum amount of demand which can be served from that facility in a period of time. The limiting factor for the capacity in practice would arise from the maximum mass of resources which can be stored at each facility, equipment and building characteristics, staffing levels etc. The agency will also assign drones to each open facility. The facilities serve as drone launching sites for distributing the resources while respecting the facility capacity and drone range constraints. In this paper, as typical in location problems, we do not consider the cost of transportation of packages and drones from warehouses to these locations. We assume 
that this cost is a constant irrespective of the configuration of the located facilities. For example, in the event of a disaster, the resources as well as drones can be airlifted to each open facility at the beginning of the planning period. We also assume that the demand during each planning horizon is smaller than the capacity of each drone. If the demand at a location is higher than the drone carrying capacity, that specific node is split into multiple nodes whose demands are less than the drone carrying capacity. Similar assumptions have been made in the drone vehicle routing problem literature (Dorling et al., 2017). Each drone makes several one-to-one trips (facility location to demand point and back) until the battery range $B$ is met as shown in Figure 1. We do not model one-to-many deliveries which require vehicle routing. This is consistent with initial applications of drone deliveries by companies such as Amazon which is focusing on single package deliveries (Amazon, 2018). As we are looking at a relatively small time frame, we do not consider recharging of drone batteries during the planning period. We assume that the drone batteries are recharged overnight or in-between planning periods. The notation used in the formulation is given below.

Figure 2 shows the demand and potential facility locations used in the case study.

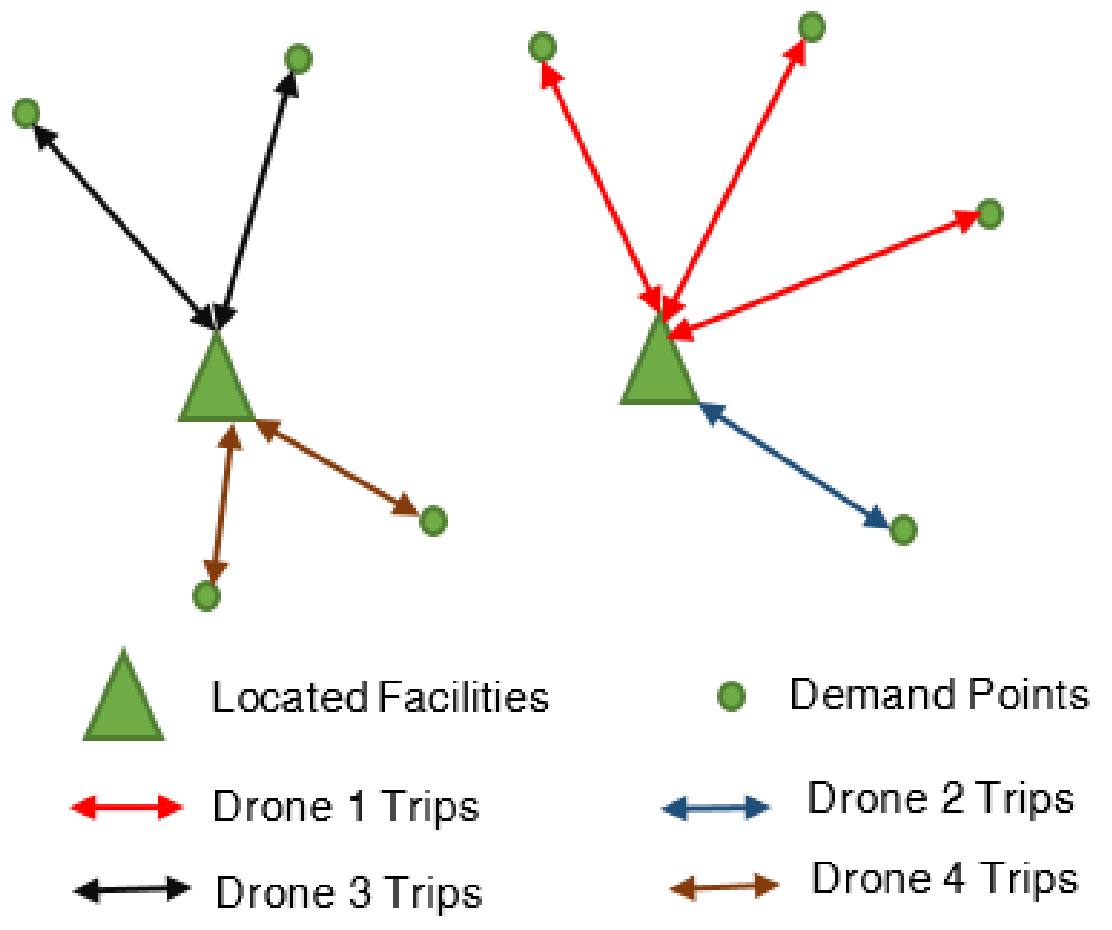

Figure 1: Schematic Representation of the Drone Delivery System

\section{Nomenclature}

\section{Sets}

I Set of all demand locations

$J \quad$ Set of all potential facility locations 


\section{Indices}

$i \in I$

$j \in J$

$k \in K$

\section{Parameters}

$\eta \quad$ Power transfer efficiency

$\theta_{s} \quad$ Lift to drag ratio

$B \quad$ Battery capacity of each drone

$b_{i j} \quad$ Battery consumed during one trip between demand $i \in I$ and facility $j \in J$

$d_{i j} \quad$ Travel distance between demand point $i \in I$ and facility $j \in J$

$m_{b} \quad$ UAV battery mass

$m_{t} \quad$ UAV mass tare, without battery and load

$p \quad$ Maximum number of facilities

$U \quad$ Capacity of each located facility (same unit as UAV mass tare and battery mass)

$w_{i} \quad$ Demand for resource at location $i \in I$ (same unit as UAV mass tare and battery mass)

\section{Decision Variables}

$x_{i j k} \quad 1$, if customer $i$ is served by the $k^{t h}$ drone of plant $j \in J$, and 0 , otherwise

$y_{j} \quad 1$, if the facility is located at $j \in J$, and 0 , otherwise

$z_{j k} \quad 1$, if the $k^{\text {th }}$ drone is assigned to facility $j \in J$, and 0 , otherwise 


$$
\begin{aligned}
& \max \sum_{i \in I} \sum_{j \in J} \sum_{k \in K} w_{i} x_{i j k} \\
& \sum_{j \in J} \sum_{k \in K} x_{i j k} \leq 1, \quad \forall i \in I \sum_{j \in J} y_{j} \leq p \\
& \sum_{i \in I} b_{i j} x_{i j k} \leq B z_{j k}, \quad \forall j \in J, k \in K \\
& \sum_{i \in I} \sum_{k \in K} w_{i} x_{i j k} \leq U y_{j}, \quad \forall j \in J \\
& z_{j k} \leq y_{j}, \quad \forall j \in J, k \in K \\
& \sum_{j \in J} z_{j k} \leq 1, \quad \forall k \in K \\
& x_{i j k}, y_{j}, z_{j k} \in\{0,1\}, \quad \forall i \in I, j \in J, k \in K
\end{aligned}
$$

The objective 1 is to maximize the demand served. Constraint 2 ensures that each demand location is covered at most once. Equation 3 restricts the number of facilities located to be less than or equal to $p$. Constraint 4 enforces battery range constraints on all the drones. Constraint 5 forces the demand served by each located facility to be less than or equal to the capacity of the facility. Constraint 6 ensures that vehicles are assigned only to located facilities. Constraint 7 ensures that each drone is assigned to at most one open facility. Constraint 8 corresponds to variable definition constraints and forces all decision variables to be binary.

The total power consumed in a delivery from facility $j \in J$ to demand point $i \in I$ is given as follows Figliozzi (2017):

$$
b_{i j}=\frac{m_{t}+m_{b}+w_{i}}{\theta_{s} \eta} d_{i j}+\frac{m_{t}+m_{b}}{\theta_{s} \eta} d_{j i} \quad \forall i \in I, j \in J
$$

In this manuscript, we do not consider the impact of charging cycles and weather (wind and temperature) on drone battery capacity. We assume that B is a point estimate which accounts for the above factors. The uncertainty associated with the daily capacity will be considered in a future work. Traditional capacitated facility location models consider location of facilities and allocation of demand points to located facilities. Joint location routing problems consider location of facilities, allocation of demand points to located facilities, and allocation of demand points to vehicle routes with multiple deliveries per route. The model studied in this paper, MCFLPD, is a new type of facility location model. In addition to the location allocation feature of traditional facility location problems, MCFLPD has drone to facility and demand to drone allocation feature while accounting for drone range restrictions. The combination of all of these features makes it a computationally complex problem to solve. The MCFLPD is a more complicated version of the maximum 
coverage capacitated facility location model which is an NP-Hard problem (Church and ReVelle, 1974; Daskin, 2011).

Traditionally, facility location decisions are long-term strategic decisions and therefore, computational performance is not that important. However, in this paper, the location decision is operational in nature. At the beginning of the time period (which is short-term like a day), an agency will know the demand patterns, weather conditions etc. and take decisions on where to open facilities (locate a pre-specified number of fully charged drones and resources of mass $U$ ) and use the drones to deliver the resources to the demand points. During the next time period, if the weather conditions and demand patterns are similar, then we can use the same solution. Otherwise, we will have to reoptimize the system quickly and potentially open new facilities and relocate the drones. For this purpose, we have developed two heuristics which are described next.

\section{Solution Approach}

This section presents two solution techniques to solve the MCFLPD problem - greedy and three-stage heuristic (3SH). The MCFLPD problem has an inherent complex knapsack structure for which greedy heuristics have been found to be efficient (Loulou and Michaelides, 1979; Goundan and Schulz, 2007; Kang and Park, 2003; Puchinger and Raidl, 2007). We hypothesized that greedy heuristic might be effective for tackling MCFLPD which belongs to the same family. The second solution procedure we developed was a decomposition based three-stage heuristic (3SH). Decomposition heuristics have been found to be useful for problems of this nature in the literature review (Kim et al., 2017; Yurek and Ozmutlu, 2018; Hong et al., 2018) and in traditional location routing problems (Wu et al., 2002; Melo et al., 2009). The final step of the 3SH procedure involves a local exchange heuristic which has been found to be efficient for complex facility location problem variants (Halper et al., 2015). We did explore different Lagrangean Relaxations by dualizing combinations of capacity, allocation, and drone range constraints. However, the bounds obtained were weak and we did not further pursue this direction. Similar insights regarding weak Lagrangean Relaxation bounds were found by Halper et al. (2015) in the context of mobile facility location problem.

\subsection{Greedy Heuristic}

The greedy heuristic has the following steps: (i) creating and sorting a weight matrix, (ii) demand allocation to open facilities, (iii) drone allocation to open facilities, and (iv) demand assignment to drones. Let $\delta_{i j}$ be an indicator variable which takes value 1 if demand point $i$ is assigned to open facility $j$ and 0 otherwise. The $\delta_{i j}$ variable is initialized to 0 for all demand points and potential facility locations.

Weight Matrix: A weight matrix $w t_{i j}$, is calculated as:

$$
w t_{i j}=\left\{\begin{array}{ll}
\frac{w_{i}}{b_{i j}} & \text { if } b_{i j} \leq B \\
0 & \text { otherwise }
\end{array} \quad \forall i \in I, j \in J\right.
$$

A demand and facility location pair with larger weights will have a higher chance of being assigned to each other. The weights are then sorted in non-increasing order and stored in a weight array. Each element 
in the weight array will have a demand location $i$ and a potential facility location $j$ associated with it. The sorted weight array is traversed sequentially, and the following demand allocation procedures are applied to each entry.

Demand Allocation: If demand $i$ is already assigned to an open facility, move to the next entry in the sorted weight array. If the demand $i$ is not assigned to any open facility, check if the associated facility $j$ is open. If facility $j$ is open, set $\delta_{i j}=1$ as long as $w t_{i j}>0$ and there is residual capacity available in facility $j$ to serve $d_{i}$. If facility $j$ is not open, open facility $j$ if the number of facilities currently open is less than $p$ and $w t_{i j}>0$ and set $\delta_{i j}=1$. If facility $j$ is not open and if we have already located $p$ facilities then identify a facility $j^{\prime}$ among the set of open facilities which has the lowest $b_{i j^{\prime}}$ and available capacity and set $\delta_{i j^{\prime}}=1$. If we are unable to assign demand $i$ to an open facility, move to the next element of the sorted weight array. Continue the demand allocation procedure until all the elements of the sorted weight array with positive weights have been processed.

Drone Allocation: Let $\hat{J}$ denote the set of all open facilities. The number of drones required to serve all demands assigned is calculated for each open facility, $N D_{j}$, as:

$$
N D_{j}=\left\lceil\sum_{i \in I} \frac{b_{i j} \delta_{i j}}{B}\right\rceil \forall j \in \hat{J}
$$

Let $\hat{J}_{o}$ represent the open facilities sorted in non-increasing order of $N D_{j}$. Traverse the set $\hat{J}_{o}$ and assign one drone to each open facility sequentially. In the first round, $p$ drones will be assigned. After assigning one drone to all open facilities, go back to the first facility in $\hat{J}_{o}$ and continue assigning one drone to each facility sequentially. If the number of drones assigned to a facility is equal to the number of drones required $N D_{j}$, then delete that facility from $\hat{J}_{o}$ and continue the drone assignment. Stop the process when all drones are assigned to open facilities or when the drone requirements of all facilities are met.

Demand to Drone Assignment: For each facility, sort the demand locations in non-decreasing order of battery consumption. Traverse the sorted demand array sequentially and assign demand locations to the first drone as long as the constraints (determined by battery consumption) are not violated. Assign the first demand location which violated the drone range constraint of the first drone to the second drone and continue assigning demands (if the facility has a second drone assigned to it). Repeat until all demands associated with that facility are assigned to drones or until it is not possible to assign any more demands to the final drone for that facility without violating the drone range constraint. Repeat the process for all facilities.

The demand coverage can be calculated by adding the demands for all locations which have been served. The greedy heuristic will ensure the facility capacity and drone range constraints are met.

\subsection{Three Stage Heuristic (3SH)}

The 3SH heuristic solves the problem in three steps. In the first stage, we solve a facility location problem and determine the facilities to be located and the demand points to be assigned to each facility. In the second 
stage, knapsack problems are solved to assign drones to facilities and demand points to drones. In the third stage, an exchange heuristic is applied to improve the solution.

\subsubsection{Facility Location and Allocation}

The following facility location allocation problem is solved to determine the facilities to be located. Let $\bar{J}_{i}$ denote the set of potential facility locations which are within the range of the drone for each demand location $i$, i.e, $\bar{J}_{i}=\left\{j: b_{i j} \leq B\right\}$. The decision variables for this optimization formulation are: (i) $\hat{x}_{i j}$ which takes value 1 if demand $i \in I$ is assigned to facility $j \in J$ and 0 otherwise, and (ii) $\hat{y}_{j}$ which takes value 1 if the facility $j$ is located and 0 otherwise.

$$
\begin{aligned}
& \max \sum_{i \in I} \sum_{j \in \bar{J}_{i}} \frac{w_{i}}{b_{i j}} \hat{x}_{i j} \\
& \sum_{j \in \bar{J}_{i}} \hat{x}_{i j} \leq 1, \quad \forall i \in I \\
& \sum_{j \in J} \hat{y}_{j} \leq p \\
& \sum_{i \in I} w_{i} \hat{x}_{i j} \leq U, \quad \forall j \in \bar{J}_{i} \\
& \hat{x}_{i j} \leq \hat{y}_{j}, \quad \forall i \in I, j \in \bar{J}_{i} \\
& \hat{x}_{i j}, \hat{y}_{j} \in\{0,1\}, \quad \forall i \in I, j \in \bar{J}_{i}
\end{aligned}
$$

Constraint 11 ensures that each demand point is assigned to at most one of the facilities within flying range. Constraint 12 enforces that at most $p$ facilities are located. Equation 13 ensures that the sum of demand assigned to a facility is less than the facility capacity. Constraint 14 makes sure that each demand point is assigned to located facility only. The objective function maximizes the weight of assigning demand points to facilities where the weight is defined in equation 9 . The above formulation is similar to a capacitated p-median facility location problem (Daskin, 2011).

\subsubsection{Repeated Application of Knapsack Problems}

Let $\hat{J}$ and $\hat{I}_{j}$ denote the set of facilities located and the set of demand locations assigned to each open facility obtained at the end of the facility location and allocation stage. Note that $\hat{J}=\left\{j \in J: \hat{y}_{j}=1\right\}$ and $\hat{I}_{j}=\left\{i: \hat{x}_{i j}=1\right\}$. In the second step, we assign drones to facilities and demand locations to drones by repeatedly solving the maximum profit knapsack problem. For any open facility $j \in \hat{J}$ and drone $k \in K$, the maximum profit knapsack problem can be defined as follows: 


$$
\begin{gathered}
C_{j}=\max \sum_{i \in \hat{I}_{j}} w_{i} x_{i}^{\prime} \\
\sum_{i \in \hat{I}_{j}} b_{i j} x_{i}^{\prime} \leq B \\
x_{i}^{\prime} \in\{0,1\}
\end{gathered}
$$

In the above formulation, the decision variable is $x_{i}^{\prime}$ which takes value 1 if demand $i$ is served by a drone and 0 otherwise. $C_{j}$ denotes the optimal objective function (16) value which corresponds to the maximum demand which can be served by a drone at a facility $j$ from the set of demand locations $\hat{I}_{j}$. Constraint 17 ensures that the demand points assigned to a drone are within the battery range, i.e., a drone can make one-to-one deliveries to all the assigned demand points without exhausting the battery capacity. The steps of the second stage of $3 \mathrm{SH}$ are described below.

- Solve $p$ maximum profit knapsack problem, one for each facility in $\hat{J}$. Let $j^{\prime}$ denote the facility with the maximum value of $C_{j}, \forall j \in \hat{J}$. Assign the first drone to facility $j^{\prime}$. Assign the demand locations in $\hat{I}_{j^{\prime}}$ with $x_{i}^{\prime}=1$ to the first drone. Update $\hat{I}_{j^{\prime}}$ by removing all demand points which have been assigned to the drone.

- Solve the maximum profit knapsack problem for facility $j^{\prime}$ with the new set of demand points $\hat{I}_{j^{\prime}}$. Update the value of $C_{j^{\prime}}$. Compare the $p$ knapsack objectives and determine the facility with a maximum value of $C_{j}$. Let $j^{\prime \prime}$ denote the facility with the highest knapsack objective. Assign the second drone to facility $j^{\prime \prime}$. Assign the demand points in $\hat{I}_{j^{\prime \prime}}$ with $x_{i}^{\prime}=1$ to the second drone. Update $\hat{I}_{j^{\prime \prime}}$ by removing all demand points which have been assigned to the drone.

- Repeat the above steps until all drones or all demand points have been assigned. The number of repetitions will be at most $|K|-1$. Now we can determine the coverage by adding the demand of all points which have been served.

The second stage involves solving at most $p+|K|-1$ maximum profit knapsack problems in total.

\subsection{3. r-Exchange Heuristic}

In the third stage, we employ a local exchange heuristic to improve the solution. First, set $\hat{J}_{0}=\hat{J}$ and determine the total demand served by each facility. The $r$ lowest demand facilities are identified and removed from the set $\hat{J}$. The set of open facilities $\hat{J}$ is then updated with $r$ facilities which are randomly picked from the remaining $|J|-p+r$ facility locations. Update the $\bar{J}_{i}=\left\{j \in \hat{J}: b_{i j} \leq B\right\}$. Now that we have fixed the open facilities and identified the facilities which can serve each demand location based on the drone range, the following allocation problem is solved. The allocation formulation shown below, equation $19-23$, is almost the same as formulation $10-15$ with one difference. In the formulation shown below $\hat{y}_{j}=1 \forall j \in \hat{J}$ 
and 0 otherwise and is not a decision variable. The decision variables are $\hat{x}_{i j}$ which take value 1 if demand $i \in I$ is assigned to facility $j \in \bar{J}_{i}$ and 0 otherwise.

$$
\begin{gathered}
\max \sum_{i \in I} \sum_{j \in \bar{J}_{i}} \frac{w_{i}}{b_{i j}} \hat{x}_{i j} \\
\sum_{j \in \bar{J}_{i}} \hat{x}_{i j} \leq 1, \quad \forall i \in I \\
\sum_{i \in I} w_{i} \hat{x}_{i j} \leq U, \quad \forall j \in \bar{J}_{i} \\
\hat{x}_{i j} \leq \hat{y}_{j}, \quad \forall i \in I, j \in \bar{J}_{i} \\
\hat{x}_{i j} \in\{0,1\}, \quad \forall i \in I, j \in \bar{J}_{i}
\end{gathered}
$$

Once the allocation problem is solved, the second stage is repeated by solving $p+|K|-1$ max profit knapsack problems. If there was an increase in the total demand served, the current best solution is recorded and $\hat{J}_{0}$ is updated to be the new set of open facilities $\hat{J}$. If there was no improvement, the current best solution corresponds to the total demand served by the open facilities $\hat{J}_{0}$ and a new set of $r$ facilities is randomly chosen. This exchange heuristic is run a pre-specified number of times.

\section{Numerical Analysis}

The feasibility of using drones for deliveries is tested on a Portland Metropolitan Area case study. The centroids of ZIP Code Tabulated Areas (ZCTAs) in the Portland Metro Region are selected as the demand locations for the case study. There are a total of 122 demand locations. The community centers throughout the Portland Metro Area are selected as the potential facility locations (refer Appendix A). In our study, the facilities should have adequate space for launching drones as well as storing the resources which are supplied. Community centers in Portland Metropolitan Area satisfy both criterions and are public facilities. There are a total of 104 potential facility locations in the case study. The demand locations and potential facility location do not overlap. Figure 2 shows the demand and potential facility locations used in the case study.

The 122 payloads to be delivered at customer demand points are randomly generated from a discrete uniform distribution ranging from $1 \mathrm{~kg}$ to $5 \mathrm{~kg}$ at intervals of $0.25 \mathrm{~kg}$ (refer Appendix A). The sum of all demands is $366.5 \mathrm{~kg}$. The capacities of each facility $U$ are generated as in Pirkul and Schilling (1989) as shown below:

$$
U=\frac{\sum_{i \in I} w_{i}}{0.8 p}
$$

In the above equation, the numerator represents the total demand to be satisfied, and $p$ denotes the number of facilities to be located. In this study, the number of facilities to be located varies from 5 to 30 in multiples of 5 . The travel distance between the demand and potential facility location is taken to be 


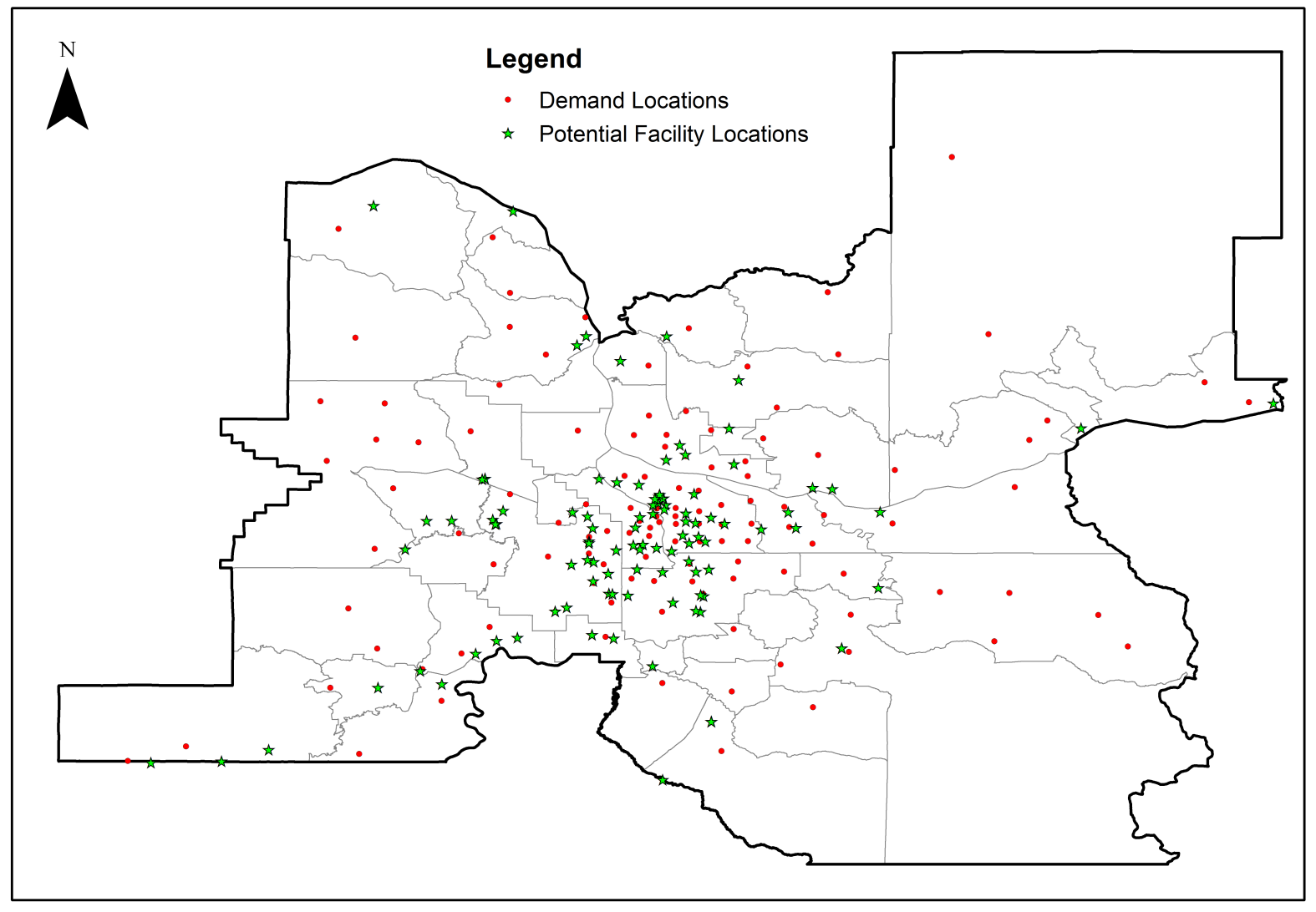

Figure 2: Demand and potential Facility Locations in the Portland Metropolitan Region

the Euclidian distance, as drones typically travel in straight lines between two points. We do not consider the impact of obstacles such as mountains or tall buildings or "no drone zones" (FAA, 2018a) in this case study. This can be a potential future extension. The following parameters are assumed for the drones unless specified otherwise (Figliozzi, 2017):

- Power transfer efficiency $(\eta) 0.66$

- Lift to drag ratio $\left(\theta_{s}\right) 3.5$

- Tare weight $10.1 \mathrm{~kg}$

- Maximum payload $5 \mathrm{~kg}$

- Battery capacity $777 \mathrm{Wh}$

- Battery safety factor 1.25 (80 \% of Maximum Battery Capacity)

All computational runs are conducted on a Windows 10 desktop with Intel Core i7-7700 CPU 3.6 GHz, 4 Core(s), 8 Logical Processor and 32 GB of RAM. 


\subsection{Computational Efficiency}

Computational efficiency of MCFLPD solutions is a key factor in real-world implementations. Initial conditions associated to customer demands and/or weather conditions (wind direction and speed) may change suddenly and may require a fast global reoptimization of the MCFLPD with different safety factors (a change in regional weather conditions will affect the energy consumption of all deliveries). A later section describes a sensitivity analysis based on different levels of allowable battery consumption. The Portland Case Study is solved using the following three methods:

- Gurobi solver using the Python interface. The model is run for a maximum of 7200 seconds or until a solution is obtained. Default parameters are assumed for the Gurobi Solver.

- Greedy Heuristic which is implemented in Python.

- Three Stage Heuristic (3SH): The facility location allocation problem in stage 1 and knapsack problems in stage 2 are solved using Gurobi. The number of facilities to be exchanged in the third stage is fixed at 2 unless specified otherwise. The exchange heuristic is run for a maximum of 100 iterations.

The first set of computational runs aim at comparing the computational efficiency of the greedy heuristic and 3SH with the Gurobi solver for different maximum number of facilities and drone availabilities (see Table 1). Since the facilities to be exchanged are picked randomly in $3 \mathrm{SH}$, we run 30 instances and report the average, minimum, and maximum computational times as well as coverage results. We limit the number of

facilities to be exchanged in the third stage of $3 \mathrm{SH}$ to just two. Coverage for the purpose of this paper is defined as the percentage of total demand met. 


\begin{tabular}{|c|c|c|c|c|c|c|c|c|c|c|c|c|c|c|c|}
\hline \multirow{3}{*}{$p$} & \multirow{3}{*}{$|K|$} & \multicolumn{4}{|c|}{ Gurobi } & \multicolumn{2}{|c|}{ Greedy } & \multicolumn{8}{|c|}{$3 \mathrm{SH}$} \\
\hline & & \multirow{2}{*}{$\begin{array}{l}\text { Time } \\
(\mathrm{sec})\end{array}$} & \multirow{2}{*}{$\begin{array}{c}\text { Coverage } \\
(\%)\end{array}$} & \multirow{2}{*}{$\begin{array}{l}\text { Gap } \\
(\%)\end{array}$} & \multirow{2}{*}{$\begin{array}{l}\text { Coverage at } \\
1800 \mathrm{sec}(\%)\end{array}$} & \multirow{2}{*}{$\begin{array}{l}\text { Time } \\
(\mathrm{sec})\end{array}$} & \multirow{2}{*}{$\begin{array}{c}\text { Coverage } \\
(\%)\end{array}$} & \multicolumn{4}{|c|}{ Time (sec) } & \multicolumn{4}{|c|}{ Coverage (\%) } \\
\hline & & & & & & & & S2 & Ave & Min & $\operatorname{Max}$ & S2 & Ave & Min & $\operatorname{Max}$ \\
\hline 5 & 20 & 7200 & 56.4 & 2.1 & 56.3 & 0.1 & 45.2 & 1.1 & 14.7 & 14.4 & 15.0 & 52.7 & 54.5 & 53.6 & 55.1 \\
\hline 5 & 25 & 7200 & 61.9 & 2.0 & 61.7 & 0.1 & 50.3 & 1.1 & 15.9 & 15.6 & 16.2 & 58.3 & 59.5 & 58.6 & 60.2 \\
\hline 5 & 30 & 7200 & 66.3 & 1.9 & 66.2 & 0.1 & 55.3 & 1.1 & 16.7 & 16.4 & 17.1 & 62.9 & 63.7 & 62.9 & 64.5 \\
\hline 5 & 35 & 7200 & 70.2 & 1.2 & 69.5 & 0.1 & 58.9 & 1.2 & 18.3 & 17.6 & 22.8 & 66.5 & 67.0 & 66.5 & 67.9 \\
\hline 5 & 40 & 7200 & 72.7 & 1.6 & 72.6 & 0.1 & 62.5 & 1.2 & 18.8 & 18.2 & 19.1 & 69.0 & 69.9 & 69.1 & 70.5 \\
\hline 10 & 20 & 7200 & 64.4 & 2.5 & 64.4 & 0.2 & 48.2 & 1.1 & 16.6 & 16.1 & 16.9 & 59.1 & 61.4 & 59.8 & 62.6 \\
\hline 10 & 30 & 7200 & 75 & 3.1 & 75 & 0.2 & 59.8 & 1.1 & 18.3 & 17.9 & 19.0 & 67.9 & 71.5 & 70.1 & 72.9 \\
\hline 10 & 40 & 7200 & 83.8 & 1.7 & 83.8 & 0.2 & 67.1 & 1.1 & 20.1 & 19.2 & 20.8 & 70.2 & 78.4 & 76.3 & 80.4 \\
\hline 15 & 30 & 7200 & 79.7 & 3.8 & 79.2 & 0.2 & 59.2 & 1.0 & 21.9 & 21.4 & 22.7 & 70.3 & 75.2 & 73.2 & 77.2 \\
\hline 15 & 45 & 7200 & 90.2 & 1.7 & 89.8 & 0.2 & 73.1 & 1.0 & 24.3 & 23.4 & 25.5 & 74.4 & 83.9 & 80.3 & 86.6 \\
\hline 15 & 60 & 7200 & 92.6 & 1.3 & 92.5 & 0.3 & 73.1 & 1.0 & 24.8 & 23.6 & 25.7 & 74.4 & 85.0 & 81.2 & 88.7 \\
\hline 20 & 20 & 7200 & 71.2 & 2.1 & 71.1 & 0.3 & 52.8 & 1.0 & 25.1 & 24.6 & 25.6 & 61.7 & 65.8 & 63.2 & 67.5 \\
\hline 20 & 40 & 7200 & 90.4 & 2.4 & 89.9 & 0.3 & 70.7 & 1.0 & 28.7 & 28.1 & 29.3 & 77.5 & 84.2 & 82.7 & 85.3 \\
\hline 20 & 60 & 320 & 93.8 & 0.0 & NA & 0.3 & 72.2 & 1.0 & 30.4 & 28.6 & 31.8 & 78.9 & 87.2 & 83.6 & 90.1 \\
\hline 20 & 80 & 36 & 93.8 & 0.0 & NA & 0.4 & 72.2 & 1.0 & 30.9 & 29.6 & 32.8 & 78.9 & 87.5 & 83.6 & 91.3 \\
\hline 25 & 25 & 7200 & 79.6 & 3.5 & 79.5 & 0.3 & 53.6 & 1.0 & 33.0 & 32.1 & 35.0 & 67.3 & 71.5 & 69.2 & 73.3 \\
\hline 25 & 50 & 337 & 93.8 & 0.0 & NA & 0.4 & 71.4 & 1.0 & 36.6 & 35.7 & 38.3 & 80.1 & 88.9 & 85.2 & 92.2 \\
\hline 25 & 75 & 27 & 93.8 & 0.0 & NA & 0.5 & 71.4 & 1.0 & 37.4 & 36.2 & 39.0 & 80.1 & 88.2 & 84.2 & 91.0 \\
\hline 25 & 100 & 43 & 93.8 & 0.0 & NA & 0.5 & 71.4 & 1.0 & 38.1 & 36.9 & 39.5 & 80.1 & 89.5 & 86.1 & 92.2 \\
\hline 30 & 30 & 7200 & 85.3 & 4.1 & 84.2 & 0.3 & 60.6 & 1.0 & 40.3 & 39.0 & 41.7 & 72.9 & 76.8 & 74.4 & 80.2 \\
\hline 30 & 60 & 23 & 93.8 & 0.0 & NA & 0.5 & 74.8 & 1.0 & 44.3 & 43.3 & 45.5 & 86.2 & 90.9 & 88.7 & 92.8 \\
\hline 30 & 90 & 31 & 93.8 & 0.0 & NA & 0.6 & 74.7 & 1.0 & 45.1 & 44.2 & 46.4 & 86.2 & 90.7 & 88.7 & 93.0 \\
\hline
\end{tabular}

$\mathrm{A}=$ Not Applicable

$\mathrm{S} 2=$ After Step 2

Table 1: Comparision of Gurobi solver, Greedy Heuristic, and 3SH 
The greedy heuristic achieves 78.01\% value of Gurobi on average (Best: $85.9 \%$ of Gurobi solution for $p=5,|K|=40$; Worst: $67.36 \%$ of Gurobi solution $p=25,|K|=25)$. The greedy heuristic provides solutions which are on average within $20 \%$ of the best Gurobi solution but takes a maximum computational time of only 0.6 seconds. According to the approximation algorithms literature in facility location, greedy and approximate algorithm solutions differ from optimal solutions in the worst case by at least $33 \%$ (Shmoys et al., 1997; Jain and Vazirani, 2001). The MCFLPD is a more complex variant of facility location problems. Therefore, we expect the worst case bounds to be weaker. However, we note empirically that all solutions are well within the worst case bounds for facility location problems. Gurobi solver successfully finds optimal solutions as resources become abundant ( $p$ increases and maximum available drones increases). Gurobi finds the true optimal solution, within the 2-hour runtime limit, in nearly one-third of the cases all of which have higher than 20 potential facility locations and at least 50 drones.

The average $3 \mathrm{SH}$ solutions are nearly $95 \%$ of the best Gurobi solutions on average. The best case is when $3 \mathrm{SH}$ achieves $96.9 \%$ of Gurobi solution for $p=5,|K|=20$ and $p=30,|K|=60$. In the worst case, $3 \mathrm{SH}$ achieves $90 \%$ of Gurobi solution for 25 potential facility locations and 25 maximum number of drones. The $3 \mathrm{SH}$ approach is significantly faster than Gurobi; the median reduction in computational time is 99.7\%. When the r-exchange heuristic is not run, i.e., the heuristic is stopped after repeated applications of the knapsack problem, the heuristic provides solutions which are on an average within $85.9 \%$ of the Gurobi solution with an average computational time of 1.2 seconds. The local search step takes an additional 26 seconds on average but helps improve the solution by another $9 \%$ making it close to the optimal solution.

Table 2 presents the average energy consumed per percent coverage which is calculated as follows:

$$
\text { Energy } / \text { Coverage }=\frac{\text { Average Battery Used } \times \text { Number of Drones Used }}{\text { Coverage }}
$$

Table 2 shows that Gurobi is competitive against 3SH when the number of facilities is less, but starts losing edge when $p$ becomes 15 or greater and progressively worsens against $3 \mathrm{SH}$ with an increase in $p$. The effect is also amplified with an increase in $|K|$, for the same $p$. This shows that $3 \mathrm{SH}$ is much more efficient in terms of drone employment to achieve a similar coverage.

\subsection{Coverage Analysis}

In this section, we analyze one scenario with low coverage and one scenario with high coverage: (i) $\mathrm{p}=$ 5 and $|K|=35$ with an optimal coverage of $70.2 \%$, and (ii) $\mathrm{p}=20$ and $|K|=60$ with an optimal coverage of $93.8 \%$. Figure 3 shows the delivery mapping of Gurobi solution for case (i). The delivery spiders are distinct and do not overlap over one another. The minimum facility utilization is $23.9 \%$ of its total capacity, and the maximum facility utilization is $87.8 \%$ of its total capacity. These results indicate that drone battery range capacity is constraining the coverage. With five facilities, most of the demand locations around the downtown region are covered which is intuitive as this is the area with the highest density. Coverage is limited in low-density areas, e.g., the Northeast region. 


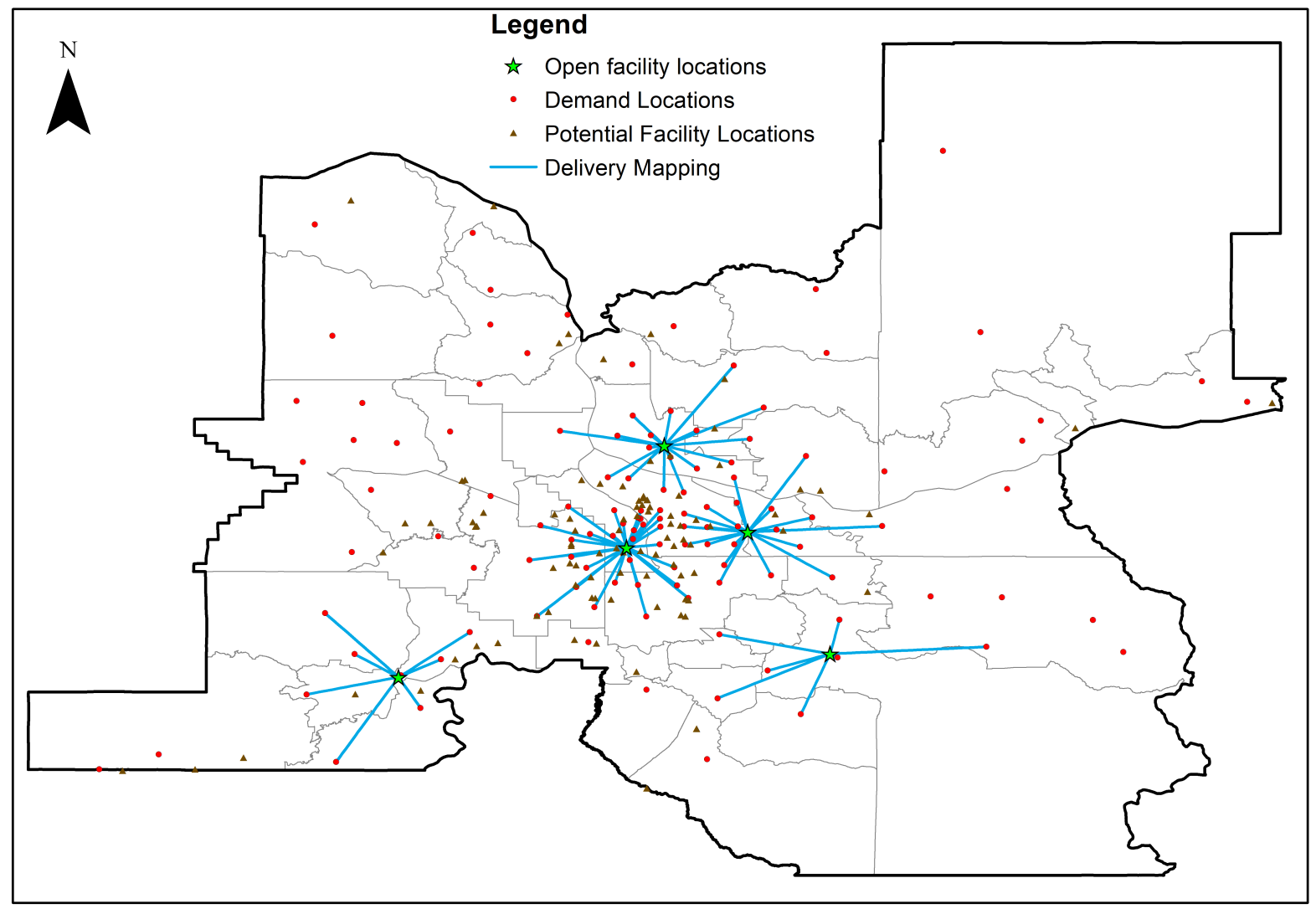

Figure 3: Case (i): Gurobi Solution $(p=5$ and $|K|=35)$ 


\begin{tabular}{c|c|c|c|c}
$p$ & $|K|$ & Gurobi & Greedy & 3 SH \\
\hline 5 & 20 & 213.9 & 205.7 & 213.3 \\
5 & 25 & 234.1 & 224.7 & 235.1 \\
5 & 30 & 259.3 & 242.7 & 257.3 \\
5 & 35 & 293.4 & 266.1 & 285.4 \\
5 & 40 & 310.5 & 291.9 & 308.2 \\
10 & 20 & 183.7 & 180.9 & 178.1 \\
10 & 30 & 226.3 & 217.3 & 227.1 \\
10 & 40 & 274.4 & 268.1 & 268.4 \\
15 & 30 & 213.7 & 219.3 & 208.8 \\
15 & 45 & 275.8 & 277.6 & 258.1 \\
15 & 60 & 347.5 & 277.6 & 268.7 \\
20 & 20 & 158.4 & 144.0 & 149.2 \\
20 & 40 & 241.7 & 233.6 & 225.7 \\
20 & 60 & 329.7 & 250.1 & 253.3 \\
20 & 80 & 386.3 & 250.1 & 253.8 \\
25 & 25 & 169.7 & 165.5 & 152.9 \\
25 & 50 & 274.4 & 249.1 & 239.7 \\
25 & 75 & 372.2 & 249.1 & 240.7 \\
25 & 100 & 416.3 & 249.1 & 242.6 \\
30 & 30 & 180.0 & 174.4 & 161.1 \\
30 & 60 & 309.5 & 248.2 & 227.5 \\
30 & 90 & 394.2 & 248.3 & 230.7
\end{tabular}

Table 2: Battery energy consumed per unit of coverage

\begin{tabular}{c|c|c} 
Unmet Demand ZCTA & Closest Facility ID & Battery Requirement $(\mathrm{Wh})$ \\
\hline 97028 & 56 & 1118 \\
97049 & 56 & 854 \\
97064 & 23 & 779 \\
97144 & 66 & 750 \\
98610 & 10 & 691 \\
98616 & 2 & 1624
\end{tabular}

Table 3: Summary of Unmet Demand Points for case (ii)

Figure 4 shows the delivery maps of Gurobi solution with 20 open facility and 60 available UAVs for case (ii). It can also be seen from the figure that the central region has a lot of overlapping spiders, which suggests that facilities have reached their capacity. The maximum facility utilization is $100 \%$ and the minimum facility utilization is $39.1 \%$ of their total capacity. Figure 5 visualizes the facilities which have utilized more than $85 \%$ of their capacity. Most of the facilities with more than $85 \%$ capacity utilization are in the downtown region. The demand points which are not served in this case are beyond the range of the drone from any community center and therefore will require improvements in battery capacity (see Table 3). The battery capacity improvements needed to achieve $100 \%$ coverage is studied in Section 5.5. The insights obtained are consistent with (Choi and Schonfeld, 2017) on the increased effectiveness of drone delivery systems in higher demand density regions and Dorling et al. (2017) on the critical nature of batteries.

We also compare the solutions obtained from 3SH and Greedy algorithm with the Gurobi solution. From Figures 5,6 , and 7 , it can be observed that facilities are more spatially dispersed in the Gurobi solution. The 
spatial dispersion shrinks a little in the 3SH solution, and then shrinks significantly in the Greedy solution, confined mostly to dense urban areas. The allocation of facilities is based on the weight matrix (equation 9). The higher the weight, the greater the priority a facility gets when the demand allocation takes place. Therefore, if a demand point and a candidate facility location are located close by (resulting in very small values of $b_{i j}$ ), the weight is higher. In the central region, the density of both, candidate facility locations and demand points, is much higher. This is evident in facility location using the Greedy algorithm (ref. Figure 6), where a majority of its open facilities are concentrated in the central region. In Figures 5, 6, and 7, the size of the stars representing open facilities indicate its capacity usage. It can be observed that the density of facilities in the central region in Gurobi solution is much lesser and also that, all of the facilities opened there have capacity utilization greater than $85 \%$. While, in the Greedy solution the facilities are "boxed-in" the urban core which results in very less capacity utilization of capacities. The capacity utilization in 3SH is $\underline{\text { much more consistent across facilities compared to Greedy solution. It can also be noticed that the number }}$ of facilities utilizing more than $85 \%$ of its capacity significantly reduced for $3 \mathrm{SH}$ and Greedy in comparison to Gurobi. In spite of this, the 3SH solution provides about 95\% coverage of the Gurobi coverage.

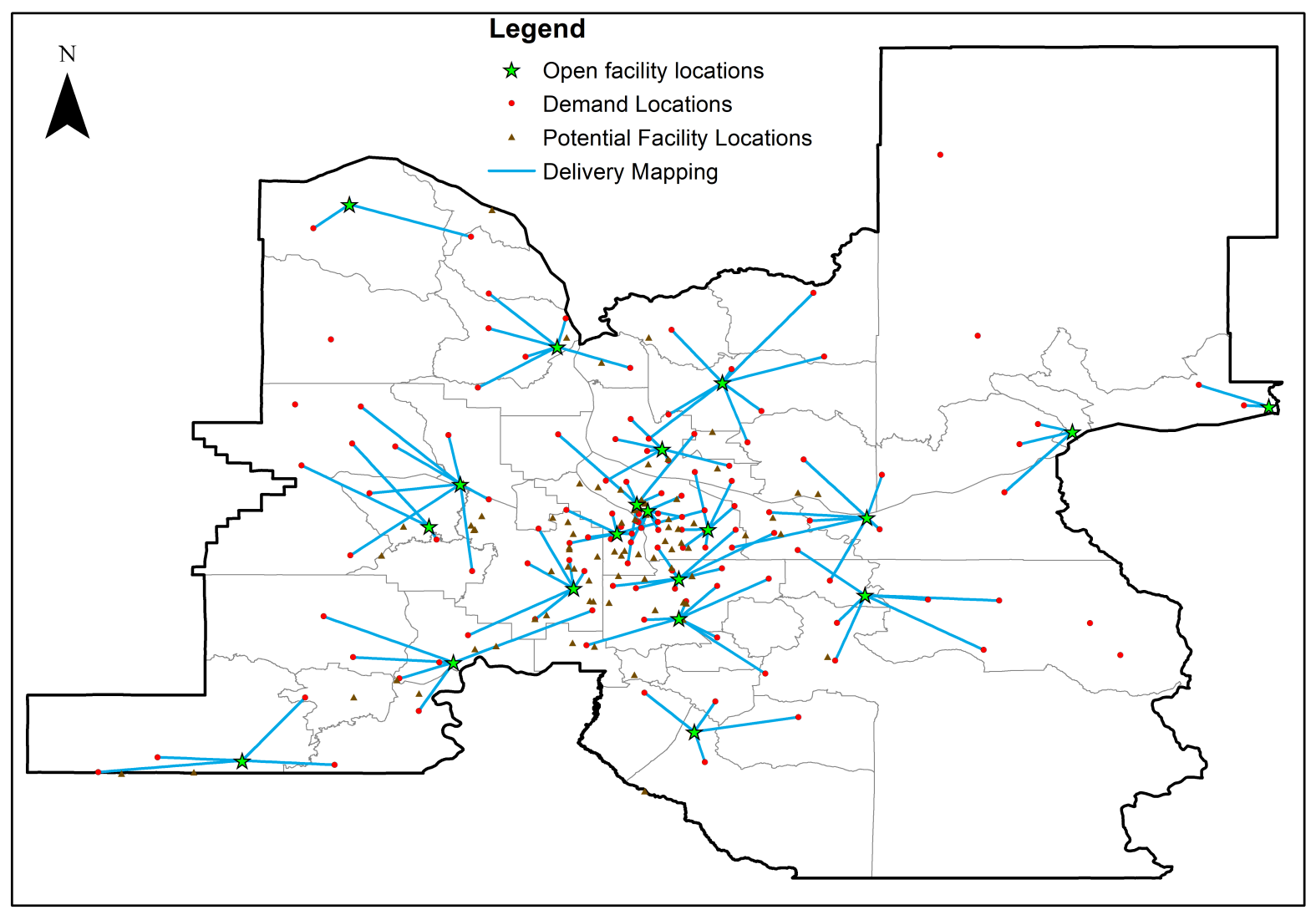

Figure 4: Case (ii): Gurobi solution $(p=20$ and $|K|=60)$ 


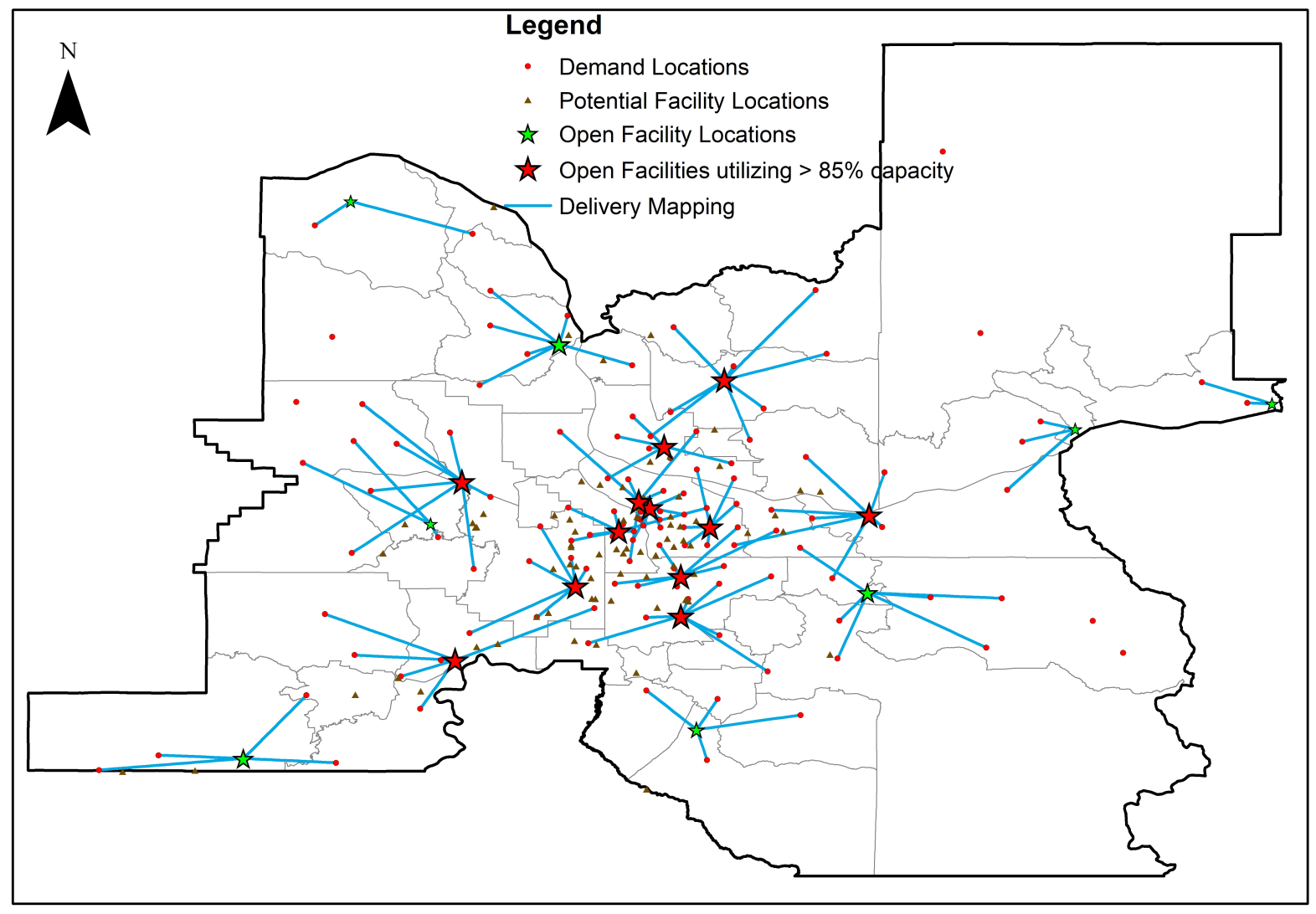

Figure 5: Case (ii): Gurobi solution highlighting facilities which have greater than $85 \%$ utilization $(p=20$ and $|K|=60)$. Size of star corresponds to facility utilization. 


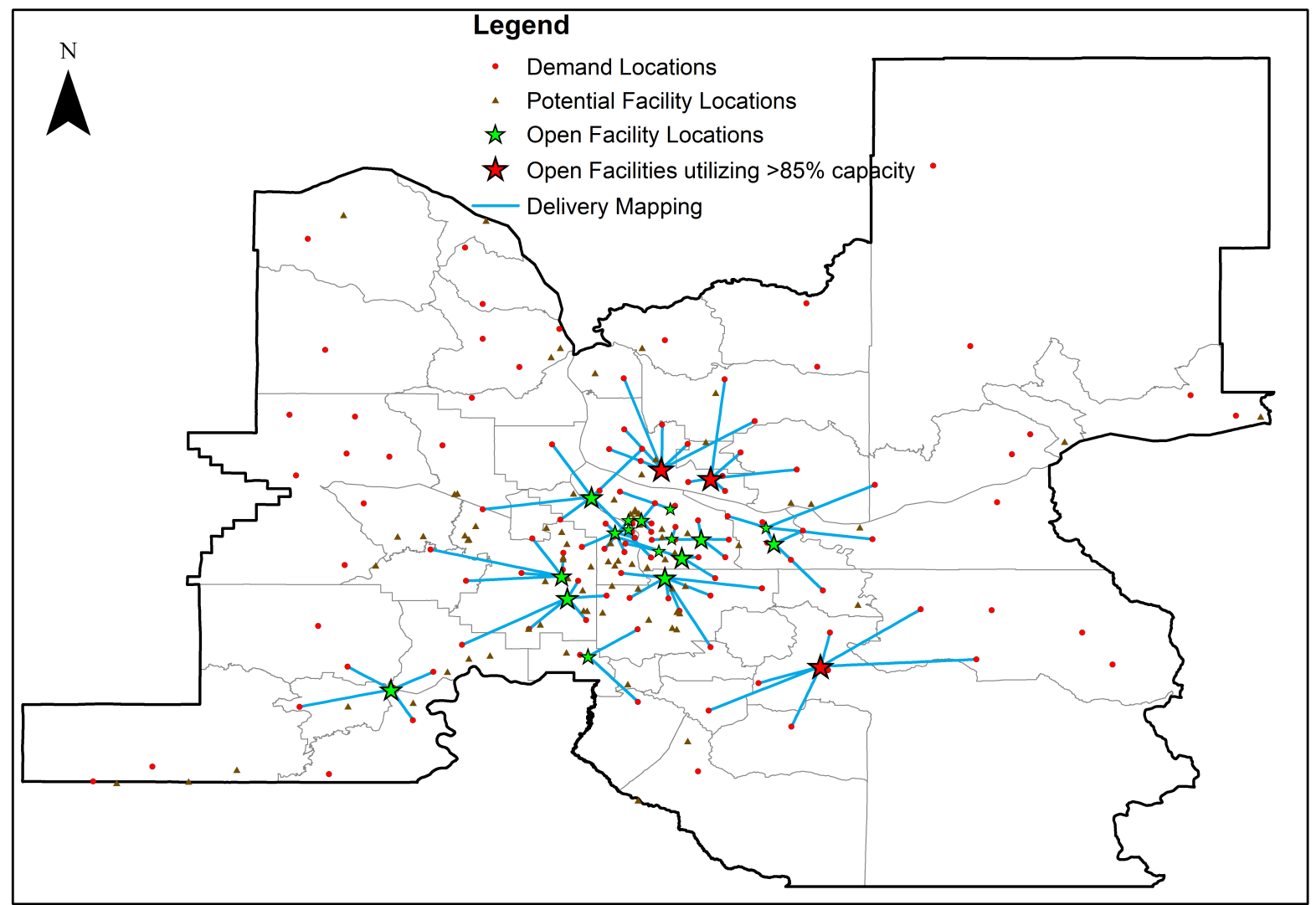

Figure 6: Greedy solution highlighting facilities which have greater than $85 \%$ utilization $(p=20$ and $|K|=60)$. Size of star corresponds to facility utilization. 


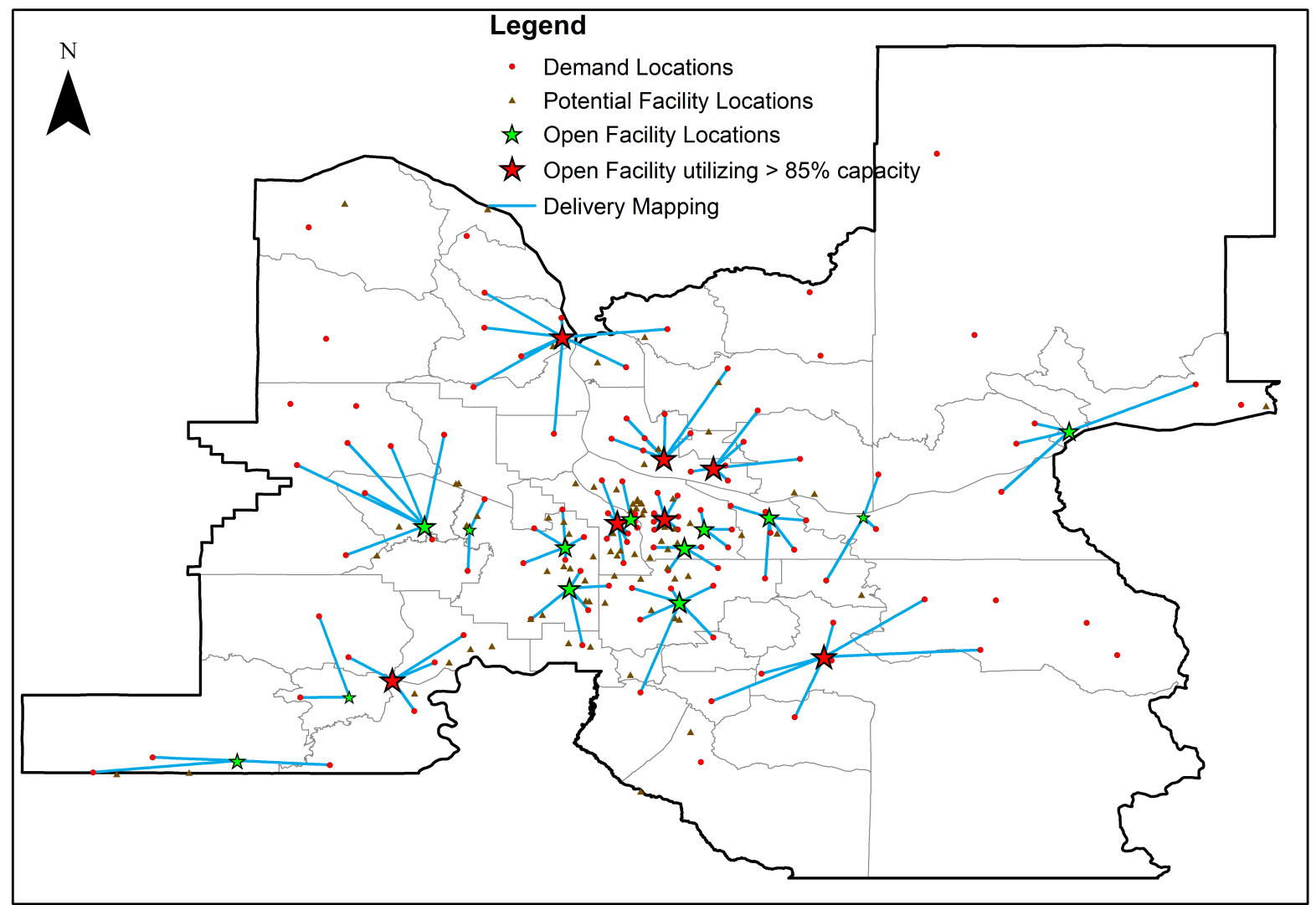

Figure 7: $3 \mathrm{SH}$ solution highlighting facilities which have greater than $85 \%$ utilization $(p=20$ and $|K|=60)$. Size of star corresponds to facility utilization. 


\begin{tabular}{c|c|c|c|c|c}
\multirow{2}{*}{$p$} & \multicolumn{5}{|c}{ Percent deviation with respect to $80 \%$ MBU } \\
\cline { 2 - 6 } & $70 \%$ MBU & $75 \%$ MBU & $85 \%$ MBU & $90 \%$ MBU & Range \\
\hline 5 & -7.5 & -3.5 & 2.1 & 4.2 & 11.8 \\
10 & -5.9 & -3.9 & 1.7 & 4.3 & 10.3 \\
15 & -4.8 & -2.1 & 1.8 & 3.9 & 8.8 \\
20 & -4.5 & -2.0 & 1.8 & 4.0 & 8.5 \\
25 & -4.2 & -1.7 & 1.1 & 3.0 & 7.3 \\
30 & -4.0 & -2.3 & 1.4 & 3.1 & 7.2
\end{tabular}

Table 4: Variation of percent deviation in average coverage with respect to the average coverage achieved at $80 \% \mathrm{MBU}$

\subsection{Sensitivity to Battery Safety Factor}

In the initial set of experiments, the battery safety factor is set to be 1.25 (drones cannot utilize more than $80 \%$ of the battery capacity) to account for weather-related uncertainties, battery usage during takeoff and landing, and uncertainties regarding initial battery conditions (Figliozzi, 2017; Microdrones, 2018). The goal of this set of experiments is to study the impact of the variation in battery safety factor on the coverage. The battery safety factor considered in this analysis include safety factors 10/7.0, 10/7.5, 10/8.5, and 10/9.0. These correspond to $70 \%, 75 \%, 85 \%$, and $90 \%$ of the battery capacity respectively. Table 4 presents the average of percentage deviation of coverage at specified maximum battery utilization (MBU) from the coverage achieved assuming a maximum battery utilization of $80 \%$ across 30 runs.

$$
\text { Percent Deviation }=\sum_{i=1}^{30} \frac{\text { Coverage }_{x}^{i}-\text { Coverage }_{80}^{i}}{\text { Coverage }_{80}^{i}} \times 100
$$

Coverage $_{80}^{i}$ represents the coverage at $80 \%$ maximum battery utilization and Coverage ${ }_{x}^{i}$ is the coverage at $x \%$ maximum battery utilization in the $i^{t h}$ run of $3 \mathrm{SH}$. In general, the effects of having a high battery safety factor (less available energy) are more profound than the effects of a low battery safety factor (more available energy). As shown in Table 4, the effect of the battery safety factor decreases marginally as p (the number of open facilities) increases.

\subsection{Sensitivity to number of facilities to be exchanged in $3 S H$}

In all the runs completed until now, two facilities (two removed and added) were exchanged in the third step of the 3SH heuristic. We now vary the number of facilities to be exchanged and study the variation in computational times and improvement in coverage. The values presented in Table 5 are the average of 30 runs. A 1 facility exchange in $3 \mathrm{SH}$ works best when $p=5$; a 2 facility exchange in $3 \mathrm{SH}$ works best when $p=10$, and a 3 facility exchange in $3 \mathrm{SH}$ works best for $p \geq 15$. No benefits were found when the number of facilities exchanged was 4 or higher. The runtimes are comparable on average which is expected given that the underlying problem sizes are not significantly altered by the size of the exchange procedure.

\subsection{Sensitivity to changes in Battery Capacity}

This section shows how coverage would improve with changes in the battery capacity of the drone. All instances were solved using Gurobi. The ideal scenario portrays the improvement in the battery without 


\begin{tabular}{|c|c|c|c|c|c|c|c|c|c|}
\hline \multirow[b]{2}{*}{$p$} & \multirow[b]{2}{*}{$|K|$} & \multicolumn{2}{|c|}{ After Step 2} & \multicolumn{2}{|c|}{1 facility exchange } & \multicolumn{2}{|c|}{2 facility exchange } & \multicolumn{2}{|c|}{3 facility exchange } \\
\hline & & $\begin{array}{c}\text { Coverage } \\
(\%)\end{array}$ & $\begin{array}{l}\text { Time } \\
(\mathrm{sec})\end{array}$ & $\begin{array}{c}\text { Coverage } \\
(\%)\end{array}$ & $\begin{array}{l}\text { Time } \\
\text { (sec) }\end{array}$ & $\begin{array}{c}\text { Coverage } \\
(\%)\end{array}$ & $\begin{array}{l}\text { Time } \\
(\mathrm{sec})\end{array}$ & $\begin{array}{c}\text { Coverage } \\
(\%)\end{array}$ & $\begin{array}{l}\text { Time } \\
(\mathrm{sec})\end{array}$ \\
\hline 5 & 20 & 52.7 & 1.1 & 54.4 & 15.4 & 54.5 & 14.7 & 53.8 & 13.5 \\
\hline 5 & 25 & 58.3 & 1.1 & 60.0 & 16.1 & 59.5 & 15.9 & 59.0 & 14.3 \\
\hline 5 & 30 & 62.9 & 1.1 & 64.5 & 17.2 & 63.7 & 16.7 & 63.3 & 15.1 \\
\hline 5 & 35 & 66.5 & 1.2 & 67.8 & 18.0 & 67.0 & 18.3 & 66.9 & 15.9 \\
\hline 5 & 40 & 69.0 & 1.2 & 70.4 & 18.8 & 69.9 & 18.8 & 69.9 & 16.6 \\
\hline 10 & 20 & 59.1 & 1.1 & 60.8 & 15.5 & 61.4 & 16.6 & 61.0 & 15.7 \\
\hline 10 & 30 & 67.9 & 1.1 & 70.4 & 17.3 & 71.5 & 18.3 & 71.2 & 17.2 \\
\hline 10 & 40 & 70.2 & 1.1 & 76.7 & 18.7 & 78.4 & 20.1 & 78.4 & 18.5 \\
\hline 15 & 30 & 70.3 & 1.0 & 74.2 & 20.8 & 75.2 & 21.9 & 75.2 & 20.5 \\
\hline 15 & 45 & 74.4 & 1.1 & 82.2 & 22.7 & 83.9 & 24.3 & 84.8 & 22.4 \\
\hline 15 & 60 & 74.4 & 1.1 & 82.4 & 22.9 & 85.0 & 24.8 & 86.2 & 22.7 \\
\hline 20 & 20 & 61.7 & 1.1 & 65.7 & 24.2 & 65.8 & 25.1 & 66.3 & 23.4 \\
\hline 20 & 40 & 77.5 & 1.1 & 83.6 & 27.4 & 84.2 & 28.7 & 84.5 & 26.2 \\
\hline 20 & 60 & 78.9 & 1.1 & 85.0 & 28.8 & 87.2 & 30.4 & 88.6 & 27.3 \\
\hline 20 & 80 & 78.9 & 1.1 & 84.9 & 29.1 & 87.5 & 30.9 & 88.9 & 27.4 \\
\hline 25 & 25 & 67.3 & 1.1 & 71.1 & 31.3 & 71.5 & 33.0 & 72.0 & 29.4 \\
\hline 25 & 50 & 80.1 & 1.1 & 87.8 & 35.3 & 88.9 & 36.6 & 90.2 & 32.2 \\
\hline 25 & 75 & 80.1 & 1.1 & 87.0 & 35.8 & 88.2 & 37.4 & 89.4 & 32.3 \\
\hline 25 & 100 & 80.1 & 1.1 & 86.6 & 36.4 & 89.5 & 38.1 & 90.2 & 32.3 \\
\hline 30 & 30 & 72.9 & 1.1 & 76.9 & 38.4 & 76.8 & 40.3 & 77.1 & 34.2 \\
\hline 30 & 60 & 86.2 & 1.1 & 89.5 & 42.8 & 90.9 & 44.3 & 91.5 & 37.0 \\
\hline 30 & 90 & 86.2 & 1.1 & 89.3 & 43.4 & 90.7 & 45.1 & 91.1 & 37.0 \\
\hline
\end{tabular}

Table 5: Impact of different number of facilities exchanged in $3 \mathrm{SH}$

an increase in the drone tare weight, which may be possible due to future breakthroughs in the battery technology. The realistic scenario assumes that we can improve the battery capacity of a drone by adding additional batteries. This results in an increase in drone tare weight which leads to increased battery consumption for the same amount of distance traveled. The UAV/drone in consideration uses Lithium polymer ( $\mathrm{LiPo}$ ) batteries. It is reported that the specific energy of LiPo batteries can go up to $275 \mathrm{Wh} / \mathrm{kg}$ (Amicell, 2018). So, for the realistic case study, the specific energy is taken to be $255 \mathrm{Wh} / \mathrm{kg}$ with $80 \% \mathrm{MBU}$. The analysis is conducted for the case when $p=20,|K|=60$. It is observed that about $165 \%$ increase in the battery capacity is required to achieve full coverage in the ideal case (see Table 6). In the realistic scenario, this increase is still not sufficient to achieve $100 \%$ coverage. This is because, the increase in distance traveled is not as high as in the ideal case due to the additional battery consumption.

An important conclusion from the sensitivity analysis is that runtime increases substantially as battery capacity increases. This is likely the result of additional complexity due to the increase of feasible options that must be analyzed by the MIP solver. Hence, the value of a high quality and yet computationally efficient heuristic like $3 S H$ is likely to increase substantially in the future. 


\begin{tabular}{c|c|c|c|c|c|c}
\multirow{2}{*}{ Battery } & \multicolumn{3}{|c|}{ Ideal } & \multicolumn{3}{c}{ Realistic } \\
\cline { 2 - 7 } & Tare Mass & Coverage(\%) & Time (sec) & Tare Mass & Coverage(\%) & Time (sec) \\
\hline 777 & 10.1 & 93.8 & 410 & 10.1 & 93.8 & 410 \\
1032 & 10.1 & 96.7 & 1233 & 11.1 & 95.7 & 667 \\
1287 & 10.1 & 97.4 & 1204 & 12.1 & 97.4 & 978 \\
1542 & 10.1 & 98.7 & 2212 & 13.1 & 97.4 & 873 \\
1797 & 10.1 & 98.7 & 2557 & 14.1 & 97.4 & 1475 \\
2052 & 10.1 & 100 & 2070 & 15.1 & 98.7 & 1377
\end{tabular}

Table 6: Impact of Battery Capacity on Coverage

\section{Conclusions}

This paper presents a novel model denoted MCFLPD for coverage-based capacitated facility location problem with drones by factoring in real-life UAV battery and weight constraints. MCFLPD is substantially more complex than traditional capacitated facility location problems. As real-world drone-based deliveries have already started being implemented in the field, it is necessary to study facility location for drones not only for economic purposes but also for social/humanitarian benefit. Drone deliveries tend to be timesensitive, e.g. medical supplies, and/or subject to unexpected changes in weather conditions. Hence, solution times are as important as solution quality.

In this research, three solution approaches are presented and compared. A state of the art MIP solver deliver high-quality solutions but requires unacceptably long running times to find feasible solutions reliably. A greedy algorithm is extremely fast, less than one second on average, but at the cost of solution quality (nearly $20 \%$ coverage loss). The three-stage heuristics (3SH) is based on decomposition and local exchange principles and on average the $3 \mathrm{SH}$ solutions are within $5 \%$ of the best Gurobi solutions but require in all cases substantially less running time (at most 46 seconds). The 3SH heuristic achieves this balanced performance by leveraging the problem structure to obtain solutions with high coverage but also more economical in terms of drone employment and in an appropriate time.

The sensitivity analysis on the battery safety factors suggests that the effects of increasing battery safety are acute. An analysis to estimate the technological improvement in the battery capacity was also performed. It showed that a breakthrough in battery technology is required to achieve one hundred percent coverage for the case study considered in this work. It was also demonstrated that MIP solver (Gurobi) solution times increase substantially as battery technology increases. This result further enhances the value of an efficient algorithm such as the proposed 3SH heuristic.

\section{References}

Agatz, N., Bouman, P., Schmidt, M., 2018. Optimization approaches for the traveling salesman problem with drone. Transportation Science.

Albareda-Sambola, M., Fernández, E., Laporte, G., 2009. The capacity and distance constrained plant location problem. Computers \& Operations Research 36 (2), 597-611. 
Albornoz, C., Giraldo, L. F., 2017. Trajectory design for efficient crop irrigation with a uav. In: 2017 IEEE 3rd Colombian Conference on Automatic Control (CCAC). IEEE, pp. 1-6.

Amazon, 2018. Amazon prime air. Accessed: May 2018.

URL https://www . amazon.com/Amazon-Prime-Air/b?ie=UTF8\&node=8037720011

Amicell, 2018. Li-polymer batteries. Accessed: May 2018.

URL http://www.amicell.co.il/batteries/rechargeable-batteries/li-polymer-batteries/

Amukele, T., Ness, P. M., Tobian, A. A., Boyd, J., Street, J., 2017. Drone transportation of blood products. Transfusion 57 (3), 582-588.

Anaya-Arenas, A. M., Renaud, J., Ruiz, A., 2014. Relief distribution networks: a systematic review. Annals of Operations Research 223 (1), 53-79.

Berner, B., Chojnacki, J., 2017. Use of drones in crop protection. In: IX International Scientific Symposium "Farm Machinery and Processes Management in Sustainable Agriculture". IEEE, pp. 46-51.

Boutilier, J. J., Brooks, S. C., Janmohamed, A., Byers, A., Buick, J. E., Zhan, C., Schoellig, A. P., Cheskes, S., Morrison, L. J., Chan, T. C., 2017. Optimizing a drone network to deliver automated external defibrillators. Circulation, 2454-2465.

Burema, H., Filin, A., 2016. Aerial farm robot system for crop dusting, planting, fertilizing and other field jobs. US Patent 9,382,003.

Carlsson, J. G., Song, S., 2017. Coordinated logistics with a truck and a drone. Management Science.

Choi, Y., Schonfeld, P. M., 2017. Optimization of multi-package drone deliveries considering battery capacity. In: 96th Annual Meeting of the Transportation Research Board, Washington, DC (Paper No. 17-05769).

Chowdhury, S., Emelogu, A., Marufuzzaman, M., Nurre, S. G., Bian, L., 2017. Drones for disaster response and relief operations: a continuous approximation model. International Journal of Production Economics $188,167-184$.

Church, R., ReVelle, C., 1974. The maximal covering location problem. In: Papers of the Regional Science Association. Vol. 32(1). Springer, pp. 101-118.

Claesson, A., Bäckman, A., Ringh, M., Svensson, L., Nordberg, P., Djärv, T., Hollenberg, J., 2017. Time to delivery of an automated external defibrillator using a drone for simulated out-of-hospital cardiac arrests vs emergency medical services. Jama 317 (22), 2332-2334.

Costa, F. G., Ueyama, J., Braun, T., Pessin, G., Osório, F. S., Vargas, P. A., 2012. The use of unmanned aerial vehicles and wireless sensor network in agricultural applications. In: Geoscience and Remote Sensing Symposium (IGARSS), 2012 IEEE International. IEEE, pp. 5045-5048. 
Daknama, R., Kraus, E., 2017. Vehicle routing with drones. eprint arXiv:1705.06431.

URL https://arxiv.org/pdf/1705.06431.pdf

Daskin, M. S., 2011. Network and discrete location: models, algorithms, and applications. John Wiley \& Sons.

Dayarian, I., Savelsbergh, M., Clarke, J.-P., 2017. Same-day delivery with drone resupply. Optimization online.

URL http://www.optimization-online.org/DB_FILE/2017/09/6206.pdf

DHL, 2014. Unmanned aerial vehicles in logistics: A dhl perspective on implications and use for the logistics industry. Accessed: May 2018.

URL http://www.dhl.com/content/dam/downloads/g0/about_us/logistics_insights/DHL_ TrendReport_UAV.pdf

Dorling, K., Heinrichs, J., Messier, G. G., Magierowski, S., 2017. Vehicle routing problems for drone delivery. IEEE Transactions on Systems, Man, and Cybernetics: Systems 47 (1), 70-85.

FAA, 2018a. Airspace restrictions. Accessed: Dec 2018.

URL https://www.faa.gov/uas/where_to_fly/airspace_restrictions/

FAA, 2018b. Faa begins drone airspace authorization expansion. Accessed: May 2018.

URL https://www.faa.gov/news/updates/?newsId=90245

Faiçal, B. S., Costa, F. G., Pessin, G., Ueyama, J., Freitas, H., Colombo, A., Fini, P. H., Villas, L., Osório, F. S., Vargas, P. A., et al., 2014. The use of unmanned aerial vehicles and wireless sensor networks for spraying pesticides. Journal of Systems Architecture 60 (4), 393-404.

Farahani, R. Z., Asgari, N., Heidari, N., Hosseininia, M., Goh, M., 2012. Covering problems in facility location: A review. Computers \& Industrial Engineering 62 (1), 368-407.

Fehrenbacher, K., 2018. A new lithium-metal battery takes flight in drones. Accessed: May 2018.

URL https://www .greentechmedia.com/articles/read/a-new-lithium-metal-battery-takes-flight-in-drones\# gs.s_bKMvI

Figliozzi, M., 2017. Lifecycle modeling and assessment of unmanned aerial vehicles (Drones) CO2e emissions. Transportation Research Part D 57, 251-261.

Giles, D. K., Billing, R., Singh, W., 2016. Performance results, economic viability and outlook for remotely piloted aircraft for agricultural spraying. Aspects of Applied Biology 132, 15-21.

Golabi, M., Shavarani, S. M., Izbirak, G., 2017. An edge-based stochastic facility location problem in uavsupported humanitarian relief logistics: a case study of tehran earthquake. Natural Hazards 87 (3), 15451565. 
Goodchild, A., Toy, J., 2017. Delivery by drone: An evaluation of unmanned aerial vehicle technology in reducing co2 emissions in the delivery service industry. Transportation Research Part D: Transport and Environment.

Goundan, P. R., Schulz, A. S., 2007. Revisiting the greedy approach to submodular set function maximization. Optimization online.

URL http://www.optimization-online.org/DB_FILE/2007/08/1740.pdf

Ha, Q. M., Deville, Y., Pham, Q. D., Hà, M. H., 2018. On the min-cost traveling salesman problem with drone. Transportation Research Part C: Emerging Technologies 86, 597-621.

Halper, R., Raghavan, S., Sahin, M., 2015. Local search heuristics for the mobile facility location problem. Computers \& Operations Research 62, 210-223.

Hassanalian, M., Abdelkefi, A., 2017. Classifications, applications, and design challenges of drones: A review. Progress in Aerospace Sciences 99, 99-131.

Holguín-Veras, J., Jaller, M., Van Wassenhove, L. N., Pérez, N., Wachtendorf, T., 2012. On the unique features of post-disaster humanitarian logistics. Journal of Operations Management 30 (7-8), 494-506.

Hong, I., Kuby, M., Murray, A. T., 2018. A range-restricted recharging station coverage model for drone delivery service planning. Transportation Research Part C: Emerging Technologies 90, 198-212.

Jain, K., Vazirani, V. V., 2001. Approximation algorithms for metric facility location and k-median problems using the primal-dual schema and lagrangian relaxation. Journal of the ACM (JACM) 48 (2), 274-296.

Kang, J., Park, S., 2003. Algorithms for the variable sized bin packing problem. European Journal of Operational Research 147(2), 365-372.

Karaca, Y., Cicek, M., Tatli, O., Sahin, A., Pasli, S., Beser, M. F., Turedi, S., 2018. The potential use of unmanned aircraft systems (drones) in mountain search and rescue operations. American Journal of Emergency Medicine 36, 585-588.

Kim, S. J., Lim, G. J., Cho, J., 2018. Drone flight scheduling under uncertainty on battery duration and air temperature. Computers \& Industrial Engineering 117, 291-302.

Kim, S. J., Lim, G. J., Cho, J., Côté, M. J., 2017. Drone-aided healthcare services for patients with chronic diseases in rural areas. Journal of Intelligent \& Robotic Systems 88 (1), 163-180.

Li, K.-R., See, K.-Y., Koh, W.-J., Zhang, J.-W., 2017. Design of 2.45 ghz microwave wireless power transfer system for battery charging applications. In: Progress in Electromagnetics Research Symposium-Fall (PIERS-FALL). IEEE, pp. 2417-2423. 
Loulou, R., Michaelides, E., 1979. New greedy-like heuristics for the multidimensional 0-1 knapsack problem. Operations Research 27(6), 1101-1114.

Mack, E., 2018. How delivery drones can help save the world. Accessed: May 2018.

URL https://www .forbes. com/sites/ericmack/2018/02/13/delivery-drones-amazon-energy-efficient-reduce\#476a63f56a87

Melo, M. T., Nickel, S., Saldanha-Da-Gama, F., 2009. Facility location and supply chain management-a review. European journal of operational research 196(2), 401-412.

Microdrones, 2018. The heavy lifting drone - md4300. Accessed: May 2018.

URL https ://www . amazon. com/Amazon-Prime-Air/b?ie=UTF8\&node=8037720011

Murray, C. C., Chu, A. G., 2015. The flying sidekick traveling salesman problem: Optimization of droneassisted parcel delivery. Transportation Research Part C: Emerging Technologies 54, 86-109.

Otto, A., Agatz, N., Campbell, J., Golden, B., Pesch, E., 2018. Optimization approaches for civil applications of unmanned aerial vehicles (uavs) or aerial drones: A survey. Networks.

URL https://doi.org/10.1002/net.21818

Ozdamar, L., 2011. Planning helicopter logistics in disaster relief. OR spectrum 33 (3), 655-672.

Pirkul, H., Schilling, D., 1989. The capacitated maximal covering location problem with backup service. Annals of Operations Research 18 (1), 141-154.

Poikonen, S., Wang, X., Golden, B., 2017. The vehicle routing problem with drones: Extended models and connections. Networks 70 (1), 34-43.

Ponza, A., 2016. Optimization of drone-assisted parcel delivery. Master's thesis, University of Padova.

Puchinger, J., Raidl, G. R., 2007. Models and algorithms for three-stage two-dimensional bin packing. European Journal of Operational Research 183 (3), 1304-1327.

Pulver, A., Wei, R., 2018. Optimizing the spatial location of medical drones. Applied Geography 90, 9-16.

Scott, J. E., Scott, C. H., 2018. Models for drone delivery of medications and other healthcare items. International Journal of Healthcare Information Systems and Informatics (IJHISI) 13 (3), 20-34.

Shmoys, D. B., Tardos, É., Aardal, K., 1997. Approximation algorithms for facility location problems. In: Proceedings of the twenty-ninth annual ACM symposium on Theory of computing. ACM, pp. 265-274.

Thiels, C., Aho, J., Zietlow, S., Jenkins, D., 2015. Use of unmanned aerial vehicles for medical product transport. Air Medical Journal 34 (2), 104-108. 
Wang, C., He, X., Liu, Y., Song, J., Zeng, A., 2016. The small single and multirotor unmanned aircraft vehicles chemical application techniques and control for rice fields in china. Aspects of Applied Biology $132,73-81$.

Wang, X., Poikonen, S., Golden, B., 2017. The vehicle routing problem with drones: Several worst-case results. Optimization Letters 11 (4), 679-697.

Wu, T.-H., Low, C., Bai, J.-W., 2002. Heuristic solutions to multi-depot location-routing problems. Computers \& Operations Research 29(10), 1393-1415.

Yanmaz, E., Quaritsch, M., Yahyanejad, S., Rinner, B., Hellwagner, H., Bettstetter, C., 2017. Communication and coordination for drone networks. In: Ad Hoc Networks. Springer, pp. 79-91.

Yanmaz, E., Yahyanejad, S., Rinner, B., Hellwagner, H., Bettstetter, C., 2018. Drone networks: Communications, coordination, and sensing. Ad Hoc Networks 68, 1-15.

Yurek, E. E., Ozmutlu, H. C., 2018. A decomposition-based iterative optimization algorithm for traveling salesman problem with drone. Transportation Research Part C: Emerging Technologies 91, 249-262. 
Appendix A. List of Demand Points and Candidate Facility Locations 


\begin{tabular}{|c|c|c|c|c|c|c|c|}
\hline ZCTA & Latitude & Longitude & Demand $(\mathrm{kg})$ & ZCTA & Latitude & Longitude & Demand $(\mathrm{kg})$ \\
\hline 97014 & 45.5829 & -122.0168 & 4.50 & 97023 & 45.2785 & -122.3232 & 3.75 \\
\hline 97019 & 45.5156 & -122.2427 & 4.00 & 97027 & 45.3856 & -122.5928 & 4.50 \\
\hline 97024 & 45.5466 & -122.4424 & 3.25 & 97028 & 45.2884 & -121.8074 & 4.75 \\
\hline 97030 & 45.5092 & -122.4336 & 1.75 & 97034 & 45.4094 & -122.6835 & 1.50 \\
\hline 97060 & 45.5313 & -122.3691 & 2.75 & 97035 & 45.4135 & -122.7252 & 2.75 \\
\hline 97080 & 45.4783 & -122.3907 & 3.75 & 97038 & 45.0954 & -122.5590 & 2.25 \\
\hline 97201 & 45.5079 & -122.6908 & 2.00 & 97042 & 45.2052 & -122.5398 & 4.25 \\
\hline 97202 & 45.4827 & -122.6444 & 4.50 & 97045 & 45.3203 & -122.5365 & 2.25 \\
\hline 97203 & 45.6035 & -122.7379 & 4.75 & 97049 & 45.3464 & -121.8624 & 2.25 \\
\hline 97204 & 45.5184 & -122.6739 & 2.25 & 97055 & 45.3888 & -122.1552 & 1.25 \\
\hline 97205 & 45.5206 & -122.7102 & 3.25 & 97067 & 45.2978 & -122.0544 & 3.25 \\
\hline 97206 & 45.4824 & -122.5986 & 3.75 & 97068 & 45.3523 & -122.6686 & 5.00 \\
\hline 97208 & 45.5287 & -122.6790 & 3.25 & 97070 & 45.3061 & -122.7731 & 2.25 \\
\hline 97209 & 45.5311 & -122.6839 & 2.25 & 97086 & 45.4452 & -122.5281 & 5.00 \\
\hline 97210 & 45.5442 & -122.7267 & 4.75 & 97089 & 45.4266 & -122.4431 & 3.00 \\
\hline 97211 & 45.5811 & -122.6373 & 4.25 & 97222 & 45.4409 & -122.6181 & 1.25 \\
\hline 97212 & 45.5442 & -122.6435 & 3.50 & 97267 & 45.4084 & -122.6129 & 2.00 \\
\hline 97213 & 45.5382 & -122.6000 & 1.25 & 98601 & 45.9434 & -122.3625 & 1.75 \\
\hline 97214 & 45.5147 & -122.6430 & 2.50 & 98604 & 45.8057 & -122.5108 & 4.00 \\
\hline 97215 & 45.5151 & -122.6006 & 2.75 & 98606 & 45.7297 & -122.4564 & 3.75 \\
\hline 97216 & 45.5139 & -122.5584 & 2.75 & 98607 & 45.6422 & -122.3800 & 3.00 \\
\hline 97217 & 45.6018 & -122.7008 & 1.75 & 98629 & 45.8766 & -122.6192 & 1.75 \\
\hline 97218 & 45.5763 & -122.6009 & 3.25 & 98642 & 45.8077 & -122.6939 & 1.75 \\
\hline 97219 & 45.4542 & -122.6985 & 1.25 & 98660 & 45.6790 & -122.7205 & 1.50 \\
\hline 97220 & 45.5500 & -122.5593 & 3.25 & 98661 & 45.6401 & -122.6250 & 3.75 \\
\hline 97221 & 45.4983 & -122.7288 & 2.50 & 98662 & 45.6885 & -122.5778 & 3.00 \\
\hline 97227 & 45.5434 & -122.6781 & 4.50 & 98663 & 45.6574 & -122.6632 & 3.00 \\
\hline 97230 & 45.5578 & -122.5053 & 1.25 & 98664 & 45.6195 & -122.5772 & 4.00 \\
\hline 97231 & 45.6876 & -122.8242 & 3.25 & 98665 & 45.6795 & -122.6606 & 1.50 \\
\hline 97232 & 45.5289 & -122.6439 & 3.50 & 98675 & 45.8285 & -122.3429 & 2.50 \\
\hline 97233 & 45.5151 & -122.5033 & 2.00 & 98682 & 45.6732 & -122.4817 & 4.50 \\
\hline 97236 & 45.4829 & -122.5098 & 5.00 & 98683 & 45.6033 & -122.5102 & 3.25 \\
\hline 97239 & 45.4924 & -122.6925 & 3.25 & 98684 & 45.6306 & -122.5148 & 4.00 \\
\hline 97266 & 45.4830 & -122.5582 & 5.00 & 98685 & 45.7152 & -122.6931 & 4.75 \\
\hline 97004 & 45.2550 & -122.4494 & 4.75 & 98686 & 45.7234 & -122.6244 & 1.25 \\
\hline 97009 & 45.4230 & -122.3328 & 1.75 & 97016 & 46.0603 & -123.2670 & 1.75 \\
\hline 97011 & 45.3871 & -122.0264 & 1.25 & 97018 & 45.8971 & -122.8106 & 2.75 \\
\hline 97013 & 45.2208 & -122.6683 & 2.00 & 97048 & 46.0448 & -122.9820 & 2.75 \\
\hline 97015 & 45.4135 & -122.5368 & 1.25 & 97051 & 45.8793 & -122.9500 & 3.75 \\
\hline 97017 & 45.1765 & -122.3897 & 1.50 & 97053 & 45.8280 & -122.8833 & 2.75 \\
\hline 97022 & 45.3467 & -122.3200 & 2.25 & 97054 & 45.9422 & -122.9496 & 1.25 \\
\hline
\end{tabular}




\begin{tabular}{c|c|c|c|c|c|c|c} 
ZCTA & Latitude & Longitude & Demand $(\mathrm{kg})$ & ZCTA & Latitude & Longitude & Demand $(\mathrm{kg})$ \\
\hline 97056 & 45.7720 & -122.9694 & 4.50 & 97116 & 45.5808 & -123.1657 & 2.00 \\
97064 & 45.8591 & -123.2355 & 4.00 & 97117 & 45.6314 & -123.2884 & 3.00 \\
97101 & 45.0902 & -123.2287 & 4.25 & 97119 & 45.4689 & -123.2002 & 3.25 \\
97111 & 45.2845 & -123.1952 & 3.75 & 97123 & 45.4402 & -122.9801 & 2.75 \\
97114 & 45.1879 & -123.0766 & 3.75 & 97124 & 45.5698 & -122.9496 & 2.25 \\
97115 & 45.2752 & -123.0395 & 3.50 & 97125 & 45.6711 & -123.1969 & 1.75 \\
97127 & 45.2461 & -123.1114 & 4.25 & 97133 & 45.6861 & -123.0227 & 3.50 \\
97128 & 45.2119 & -123.2822 & 4.25 & 97140 & 45.3531 & -122.8659 & 4.50 \\
97132 & 45.3242 & -122.9873 & 5.00 & 97144 & 45.7416 & -123.3002 & 2.25 \\
97148 & 45.3584 & -123.2485 & 3.75 & 97223 & 45.4403 & -122.7766 & 3.75 \\
97347 & 45.0771 & -123.6564 & 1.50 & 97224 & 45.4055 & -122.7951 & 1.75 \\
97396 & 45.1040 & -123.5490 & 3.75 & 97225 & 45.5016 & -122.7700 & 2.00 \\
97005 & 45.4910 & -122.8036 & 3.25 & 97229 & 45.5510 & -122.8093 & 2.00 \\
97006 & 45.5170 & -122.8598 & 3.25 & 98605 & 45.7769 & -121.6655 & 4.25 \\
97007 & 45.4543 & -122.8796 & 2.00 & 98610 & 45.8659 & -122.0652 & 4.75 \\
97008 & 45.4602 & -122.8042 & 2.25 & 98616 & 46.1933 & -122.1329 & 4.75 \\
97062 & 45.3693 & -122.7623 & 1.75 & 98639 & 45.6699 & -121.9897 & 1.75 \\
97106 & 45.6657 & -123.1190 & 3.75 & 98648 & 45.7063 & -121.9563 & 2.75 \\
97109 & 45.7378 & -123.1812 & 3.00 & 98651 & 45.7399 & -121.5835 & 3.50 \\
97113 & 45.4972 & -123.0443 & 1.50 & 98671 & 45.6144 & -122.2384 & 1.50
\end{tabular}

Table A.7: List of demand points with assumed demand 


\begin{tabular}{|c|c|c|c|c|c|c|c|c|}
\hline ID & Latitude & Longitude & ID & Latitude & Longitude & ID & Latitude & Longitude \\
\hline 0 & 45.8169 & -122.7459 & 35 & 45.4831 & -122.8041 & 70 & 45.3608 & -122.8451 \\
\hline 1 & 45.8625 & -122.6605 & 36 & 45.5167 & -122.6071 & 71 & 45.4316 & -122.7151 \\
\hline 2 & 45.7812 & -122.5273 & 37 & 45.5625 & -122.6666 & 72 & 45.3535 & -122.8665 \\
\hline 3 & 45.6920 & -122.5452 & 38 & 45.5986 & -122.7853 & 73 & 45.4091 & -122.7963 \\
\hline 4 & 45.6610 & -122.6360 & 39 & 45.5662 & -122.6746 & 74 & 45.4824 & -122.5887 \\
\hline 5 & 45.6259 & -122.5361 & 40 & 45.5502 & -122.6619 & 75 & 45.4401 & -122.8366 \\
\hline 6 & 45.6335 & -122.6613 & 41 & 45.5076 & -122.4215 & 76 & 45.5074 & -122.7971 \\
\hline 7 & 45.6431 & -122.6255 & 42 & 45.5206 & -123.1042 & 77 & 45.5696 & -122.6736 \\
\hline 8 & 45.5820 & -122.3905 & 43 & 45.5051 & -122.4856 & 78 & 45.4682 & -122.7100 \\
\hline 9 & 45.5797 & -122.3542 & 44 & 45.4266 & -122.6061 & 79 & 946 & -122.6305 \\
\hline 10 & 45.6923 & -121.8941 & 45 & 45.4312 & -122.5822 & 80 & & -122.7179 \\
\hline 11 & 45.7380 & -121.5386 & 46 & 45.4771 & -122.7040 & 81 & & -122.5778 \\
\hline 12 & 45.4682 & -123.1438 & 47 & 45.4913 & -122.6014 & 82 & 5199 & -122.6246 \\
\hline 13 & 45.5987 & -122.9961 & 48 & 45.3036 & -122.7586 & 83 & 5503 & -122.6857 \\
\hline 14 & 45.2993 & -122.9750 & 49 & 45.3828 & -122.7321 & 84 & 45.5422 & -122.6649 \\
\hline 15 & 45.3046 & -122.9365 & 50 & 45.3863 & -122.7670 & 85 & 45.4758 & -122.7221 \\
\hline 16 & 45.0740 & -123.6141 & 51 & 45.3856 & .7605 & 86 & 1487 & -122.8064 \\
\hline 17 & 45.2125 & -123.1940 & 52 & 45.4233 & & 87 & 618 & -122.6819 \\
\hline 18 & 45.2432 & -123.1160 & 53 & 45.5368 & .4358 & 88 & & -122.6060 \\
\hline 19 & 45.2753 & & 54 & & & 89 & & -122.9748 \\
\hline 20 & 45.2191 & & 55 & & & 90 & 710 & -122.6790 \\
\hline 21 & 45.0979 & -123.3960 & 56 & & & 91 & & -122.9769 \\
\hline 22 & 45.0765 & -123.4832 & 57 & 450 & -122.7952 & 92 & 278 & -122.7097 \\
\hline 23 & 46.1034 & -123.2021 & 58 & & -122.8065 & 93 & 877 & -122.7114 \\
\hline 24 & 46.0933 & & 59 & & -122.6865 & 94 & 6158 & -122.5532 \\
\hline 25 & 45.8626 & -122.8090 & 60 & 45.5987 & -122.9961 & 95 & 45.5268 & -122.5785 \\
\hline 26 & 45.8459 & -122.8259 & 61 & 45.3525 & -122.5980 & 96 & 45.4648 & -122.6513 \\
\hline 27 & 45.5372 & -122.2658 & 62 & 45.3816 & -122.5923 & 97 & 45.5922 & -122.7522 \\
\hline 28 & 45.2852 & -122.3368 & 63 & 45.4461 & -122.6192 & 98 & 45.4798 & -122.6188 \\
\hline 29 & 45.0424 & -122.6677 & 64 & 45.2852 & -122.3368 & 99 & 45.4262 & -122.6679 \\
\hline 30 & 45.1499 & -122.5777 & 65 & 45.3838 & -122.5976 & 100 & 45.3699 & -122.6488 \\
\hline 31 & 45.5346 & -122.6247 & 66 & 45.5976 & -123.0012 & 101 & 45.4798 & -122.8040 \\
\hline 32 & 45.5708 & -122.6097 & 67 & 45.5623 & -122.6676 & 102 & 45.5396 & -122.9626 \\
\hline 33 & 45.5537 & -122.6759 & 68 & 45.4666 & -122.7535 & 103 & 45.3102 & -122.7982 \\
\hline 34 & 45.5339 & -122.6854 & 69 & 45.5229 & -122.9820 & & & \\
\hline
\end{tabular}

Table A.8: List of all candidate facility locations 